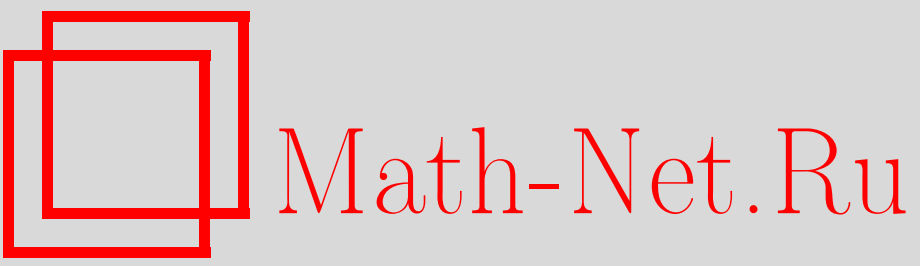

А. В. Ерохин, Ф. М. Малышев, А. Е. Тришин, Многомерный линейный метод и показатели рассеивания линейной среды шифрпреобразований, Матем. вопр. криптогр., 2017, том 8, выпуск 4, 29-62

DOI: https://doi.org/10.4213/mvk235

Использование Общероссийского математического портала Math-Net.Ru подразумевает, что вы прочитали и согласны с пользовательским соглашением

http://www . mathnet.ru/rus/agreement

Параметры загрузки:

IP: 52.90 .164 .192

26 апреля 2023 г., 17:30:30 
МАТЕМАТИЧЕСКИЕ ВОПРОСЫ КРИПТОГРАФИИ

2017 T. 8 № 4 C. 29-62

УДК 519.719.2

\title{
Многомерный линейный метод и показатели рассеивания линейной среды шифрпреобразований
}

\author{
А. В. Ерохин $^{1}$, Ф. М. Малышев ${ }^{2}$, А. Е. Тришин ${ }^{3}$ \\ 1 Лаборатории ТВП, Москва \\ ${ }^{2}$ Математический институт им. В. А. Стеклова РАН, Москва \\ ${ }^{3}$ ООО «Центр сертификаиионных исследований», Москва
}

Получено 20.IV.2017

Аннотация. Дано описание многомерного линейного метода криптографического анализа шифрпреобразований, в которых наложение ключевых бит происходит покомпонентно по $\bmod 2$. Устанавлена его связь с обычным (одномерным) линейным методом. Уточняются понятия показателей рассеивания линейной среды и линейных преобразований, используемых в алгоритмах блочного шифрования.

Ключевые слова: криптографический анализ, линейный метод, линейная среда преобразования, показатели рассеивания

Multidimensional linear method and diffusion characteristics of linear medium of ciphering transform

A. V. Erokhin ${ }^{1}$, F. M. Malyshev ${ }^{2}$, A.E. Trishin ${ }^{3}$

${ }^{1}$ TVP Laboratories, Moscow

${ }^{2}$ Steklov Mathematical Institute of RAS, Moscow

${ }^{3}$ Certification Research Center, LLC, Moscow

Abstract. We describe a multidimensional method of cryptoanalysis of ciphering transforms with key bits addition performed componentwise mod 2. It is compared with the usual (one-dimensional) method. The notions of diffusion characteristics of linear medium and linear transforms used in block ciphers are refined.

Key words: cryptoanalysis, linear method, linear medium of transform, diffusion characteristics

Citation: Mathematical Aspects of Cryptography, 2017, v. 8, № 4, pp. 29-62 (Russian)

(c) Академия криптографии Российской Федерации, 2017 г. 


\section{Введение}

После успешного применения линейного (одномерного) метода криптографического анализа к алгоритму шифрования DES [1] стали появляться усовершенствования этого метода. Одно усовершенствование связано с использованием нескольких линейных (одномерных) соотношений [2-5]. Другое направление относится к многомерному ( $s$-мерному при $s>1)$ линейному методу, когда (например, в случае блочных шифров с блоками открытого и шифрованного текстов из $\left.V_{n}=G F(2)^{n}\right)$ сравниваются значения линейных отображений $V_{n} \rightarrow V_{s}$, а не линейных функций $V_{n} \rightarrow G F(2)$, как это имеет место в обычном линейном методе (при $s=1$ ). Одной из первых в этом направлении является работа [6]. Развитие и применения многомерного линейного метода содержатся в [7-10].

Для получения $s$-мерных вероятностных линейных соотношений полезно отдельные операции шифрпреобразования располагать последовательно сверху вниз. Отдельные операции (отображения) будем представлять стрелками, направленными сверху вниз. Перед каждой стрелкой (в конце предшествующей ей стрелки) располагается текущее состояние преобразуемых данных в виде булева вектора соответствующего размера. В самом верху располагается входной подлежащий шифрпреобразованию вектор. В самом низу располагается результат шифрпреобразования в виде булева вектора, возможно, другого размера. В случае блочного шифрования эти размеры одинаковы, но размеры промежуточных векторов могут быть бо́льшими.

Криптографический анализ шифрпреобразования $s$-мерным линейным методом предполагает построение линейных (над полем $G F(2)$ ) отображений в векторное пространство $V_{s}$, применяемых к входным, выходным и промежуточным булевым векторам, расположенным между вертикальными стрелками. (У нас $s>1$, в линейном методе $s=1$.) Эти линейные отображения будем представлять горизонтальными стрелками, направленными слева направо, и называть горизонтальными. Число горизонтальных стрелок на единицу больше числа вертикальных стрелок. Значения горизонтальных линейных отображений (векторы из $V_{s}$ ) естественно считать откликами промежуточной информации в шифрпреобразовании (включающей входные и выходные данные). Горизонтальные стрелки (как и отклики) располагаются одна под другой. Последовательность горизонтальных линейных отображений должна обеспечивать заметную корреляционную зависимость между соседними откликами. Данные отклики должны даже совпадать, если между соответствующими соседними горизонтальными отображениями располагаются только линейные (вертикальные) операции шифрпреобразования. 
Линейный метод вместе с двойственным ему (см. [11]) разностным методом привел к появлению новых классов алгоритмов блочного шифрования, среди которых необходимо выделить класс $X S L$-схем. Этому классу, в частности, принадлежат стандарты блочного шифрования AES и Кузнечик. Дело в том, что линейный и разностный методы позволили глубже осмыслить введенное К. Шенноном [12] качественное понятие рассеивания. Степень рассеивания стала измеряться численной характеристикой, названной показателем рассеивания линейной среды шифрпреобразования [13]. Ввиду этого разработка шифрпреобразований начинается с формирования их структуры в виде линейной среды с гарантированными показателями рассеивания, обеспечивающими защиту от линейного и разностного методов. Необходимый уровень криптографической стойкости как относительно методов линейного типа (включая разностный), так и иных методов обеспечивается затем путем специального тщательного подбора встраиваемых в линейную среду локальных нелинейных отображений.

Относительно просто оцениваемые показатели рассеивания явились удачным дополнением к таким параметрам, как длина криптографического ключа и число итераций в шифрпреобразованиях, по которым можно судить о стойкости алгоритмов шифрования.

Работа состоит из трех разделов. В первом разделе излагается $s$-мерный линейный метод, где $s \geqslant 1$, для произвольных функциональных схем, задающих отображения двоичных векторных пространств, аналогично тому, как это было в работах [11] и [13]. Во втором разделе устанавливается связь линейных методов при $s>1$ и при $s=1$. В третьем разделе (в связи с многомерным линейным методом) рассматриваются понятия показателей рассеивания линейной среды шифрпреобразований и отдельных невырожденных матриц.

\section{1. Многомерный линейный метод}

Главным в многомерном линейном методе является построение $s$-мерных вероятностных линейных соотношений, связывающих знаки открытого и шифрованного текстов. Основные приемы такого построения будут изложены в общем виде для шифрпреобразований, заданных функциональными схемами. Далее приводятся необходимые в дальнейшем понятия, относящиеся к функциональным схемам.

\section{1. Функциональные схемы}

Функциональная схема, реализующая шифрпреобразование

$$
F: V_{N} \times V_{K} \rightarrow V_{M}, \quad F(a, z)=b,(a, z) \in V_{N} \times V_{K},
$$

во многом повторяет последовательность команд программной реализации соответствующего шифратора. 
В шифрпреобразовании (1) через $V_{K}=G F(2)^{K}$ обозначается множество возможных ключей. Имеются в виду рабочие ключи. Они могут состоять, например, из последовательности бит всех раундовых ключей блочного шифратора, предварительно вырабатываемых из основного криптографического ключа. Векторы из $V_{N}$ в (1) составлены из знаков открытого текста, включая сопровождающую его дополнительную информацию, участвующую в процессе шифрования. Векторы из $V_{M}$ состоят из бит шифрованного текста. Как обычно, векторы из $V_{n}=G F(2)^{n}, n \in \mathbb{N}$, представляем вектор-строками.

В случае блочных шифров $M=N$. Тогда $a \in V_{N}-$ блок открытого текста, $b \in V_{M}$ - блок шифрованного текста, $z \in V_{K}-$ рабочий ключ. Компоненты векторов $(a, z) \in V_{N} \times V_{K}$ и $b \in V_{M}$ являются, соответственно, входами и выходами функциональной схемы.

В функциональной схеме, реализующей шифрпреобразование (1), отдельно выделяются функциональные элементы общего вида - отображения

$$
f_{i}: V_{n_{i}} \rightarrow V_{m_{i}}, \quad i=1,2, \ldots, k .
$$

Как правило, эти отображения нелинейные. Аргументы (входы) отображений $f_{i}$ обозначаем через $x_{i} \in V_{n_{i}}$, а образы (выходы) обозначаем через $y_{i} \in V_{m_{i}}, y_{i}=f_{i}\left(x_{i}\right), i=1,2, \ldots, k$. Остальные операции функциональной схемы являются линейными. Все вместе они образуют линейную среду шифрпреобразования. Среди отображений (2) общего вида могут быть и линейные, но они уже не включаются в линейную среду.

Совокупность отображений (2) можно упорядочить так, что выходы с $f_{i}$ могут поступать на входы $f_{j}$ (возможно, опосредованно через линейные операции) только при $i<j$. При $j \leqslant i$ аргументы $f_{j}$ от выходов с $f_{i}$ не будут зависеть. Тогда для $j=1,2, \ldots, k$

$$
\begin{aligned}
x_{j} & =c_{j}\left(a, z, y_{1}, \ldots, y_{j-1}\right)=a c_{0 j}+z c_{* j}+y_{1} c_{1 j}+\ldots+y_{j-1} c_{j-1, j}, \\
b & =c_{k+1}\left(a, z, y_{1}, \ldots, y_{k}\right)=a c_{0, k+1}+z c_{*, k+1}+y_{1} c_{1, k+1}+\ldots+y_{k} c_{k, k+1},
\end{aligned}
$$

где

$$
c_{j}: V_{N+K+\sum_{i=1}^{j-1} m_{i}} \rightarrow V_{n_{j}}, \quad j=1,2, \ldots, k, k+1, n_{k+1}=M,
$$

- линейные (над полем $G F(2)$ ) отображения, задаваемые матрицами $\left(\begin{array}{c}c_{0 j} \\ c_{* j} \\ c_{1 j} \\ \vdots \\ c_{j-1, j}\end{array}\right)$.

Линейные отображения и их матрицы обозначаются в работе одинаковыми символами. 
Согласно (3), (4) линейные комбинации бит ключа прибавляются к промежуточным значениям шифрпреобразования покомпонентно по $\bmod 2$. Матричные блоки $c_{i j}, 0 \leqslant i \leqslant j-1, j=1,2, \ldots, k, k+1$, имеют размер $m_{i} \times n_{j}$, где $m_{0}=N, n_{k+1}=M$. Блоки $c_{* j}, j=1,2, \ldots, k, k+1$, имеют размер $K \times n_{j}$. Линейные отображения (5) естественным образом объединяются в одно линейное отображение

$$
C: V_{N+K+\sum_{i=1}^{k} m_{i}} \rightarrow V_{\sum_{i=1}^{k} n_{i}+M}
$$

с матрицей

$$
C=\left(\begin{array}{ccccccc}
c_{01} & c_{02} & c_{03} & \ldots & c_{0, k-1} & c_{0 k} & c_{0, k+1} \\
c_{* 1} & c_{* 2} & c_{* 3} & \ldots & c_{*, k-1} & c_{* k} & c_{*, k+1} \\
0 & c_{12} & c_{13} & \ldots & c_{1, k-1} & c_{1 k} & c_{1, k+1} \\
\ldots & \ldots & \ldots & \ldots & \ldots & \ldots & \ldots \\
0 & 0 & 0 & \ldots & 0 & c_{k-1, k} & c_{k-1, k+1} \\
0 & 0 & 0 & \ldots & 0 & 0 & c_{k, k+1}
\end{array}\right)
$$

размера $\left(N+K+\sum_{i=1}^{k} m_{i}\right) \times\left(\sum_{i=1}^{k} n_{i}+M\right)$. Оно позволяет равенства (3) и (4) записать в виде $\left(a, z, y_{1}, \ldots, y_{k}\right) C=\left(x_{1}, \ldots, x_{k}, b\right)$ или, полагая $\left(y_{1}, \ldots, y_{k}\right)=y,\left(x_{1}, \ldots, x_{k}\right)=x$, в виде

$$
(a, z, y) C=(x, b) .
$$

Матрицу линейной среды $C$ разобьем на три подматрицы, состоящие из $N, K$ и $\sum_{i=1}^{k} m_{i}$ строк: $C=\left(\begin{array}{l}C_{1} \\ C_{0} \\ C_{2}\end{array}\right)$. Обозначим $\widetilde{C}=\left(\begin{array}{l}C_{1} \\ C_{2}\end{array}\right)$. Тогда условие (7) примет вид $(a, y) \widetilde{C}+z C_{0}=(x, b)$.

Представление конкретного шифрпреобразования функциональной схемой не однозначно, оно определяется выделением в нем функциональных элементов (2). По усмотрению криптоаналитика они могут быть укрупненными, что упрощает задаваемую отображением (6) линейную среду, или, напротив, предельно упрощенными, из-за чего увеличивается их число $k$ и усложняется линейная среда $C$.

Функциональные элементы (2) легче поддаются непосредственному анализу в случае небольших значений $n_{i}, m_{i}$. В тех случаях, когда непосредственный анализ отображений (2) не удается проводить из-за больших значений $n_{i}, m_{i}$, отображение $f_{i}: V_{n_{i}} \rightarrow V_{m_{i}}$ тоже приходится представлять функциональной схемой. 


\section{2. Аддитивный способ построения многомерных линейных вероятностных соотношений}

Излагаемая ниже общая схема получения многомерных вероятностных линейных соотношений та же, что и при $s=1$; она представлена в $[11,13]$. Далее считаем $s \geqslant 1$. Многомерное вероятностное линейное соотношение для шифрпреобразования (1) задается тройкой линейных отображений $L^{\prime}: V_{N} \rightarrow V_{s}, L: V_{K} \rightarrow V_{s}, L^{\prime \prime}: V_{M} \rightarrow V_{s}$ и представляется в виде

$$
b L^{\prime \prime}=a L^{\prime}+z L+\eta,
$$

где $b=F(a, z), \quad \eta=\eta(a, z) \in V_{s}-$ случайный вектор «невязки», если $a \in V_{N}-$ случайный вектор, а $z \in V_{K}-$ фиксированный ключ. Вероятностное распределение вектора $a$ на $V_{N}$ будем предполагать равномерным. Матрица $L^{\prime \prime}$ в выражении (8) выбирается ненулевой.

Эффективность соотношения (8) характеризуется распределением вектора $\eta$ на $V_{s}$. Чем ближе это распределение к вырожденному (сосредоточенному в одной точке) и чем дальше от равномерного распределения, тем эффективнее соотношение (8). В качестве меры неопределенности для вектора $\eta$ рассматривается энтропия $H(\eta)$ его распределения на $V_{s}$ [12].

Многомерные вероятностные линейные соотношения (8) интересны не сами по себе, а прежде всего как средство для определения ключа $z \in V_{K}$ по известным парам $\left(a^{(j)}, b^{(j)}\right) \in V_{N} \times V_{M}, j=1,2, \ldots, T$.

Соотношение (8) предлагается получать путем суммирования аналогичных так называемых локальных $s$-мерных вероятностных линейных соотношений для отдельных отображений (2) при всех $i=1,2, \ldots, k$, записываемых в виде

$$
y_{i} l_{i}^{\prime \prime}=x_{i} l_{i}^{\prime}+\eta_{i},
$$

где $y_{i}=f_{i}\left(x_{i}\right), \eta_{i}=\eta_{i}\left(x_{i}\right) \in V_{s}-$ случайный вектор «невязки», если $x_{i} \in V_{n_{i}}$ - случайный вектор.

Вся совокупность соотношений (9) задается набором

$$
\mathfrak{L}=\left(\left(l_{i}^{\prime}, l_{i}^{\prime \prime}\right), i=1,2, \ldots, k\right) \quad \text { или } \quad \mathfrak{L}=\left(l^{\prime}, l^{\prime \prime}\right), \quad l^{\prime}=\left(\begin{array}{c}
l_{1}^{\prime} \\
\vdots \\
l_{k}^{\prime}
\end{array}\right), l^{\prime \prime}=\left(\begin{array}{c}
l_{1}^{\prime \prime} \\
\vdots \\
l_{k}^{\prime \prime}
\end{array}\right),
$$

состоящим из двоичных матриц размеров $n_{i} \times s$ и $m_{i} \times s$, отвечающих линейным отображениям $l_{i}^{\prime}: V_{n_{i}} \rightarrow V_{s}, l_{i}^{\prime \prime}: V_{m_{i}} \rightarrow V_{s}, i=1,2, \ldots, k$. Предполагается, что множество всех строк матриц $l_{i}^{\prime}, \quad l_{i}^{\prime \prime}, i=1,2, \ldots, k$, мощности $\sum_{i=1}^{k}\left(n_{i}+m_{i}\right)$, порождает все пространство $V_{s}$. 
К отображениям $l_{i}^{\prime}, l_{i}^{\prime \prime}, i=1,2, \ldots, k$, предъявляются два требования.

Первое требование относится к отдельным парам отображений $\left(l_{i}^{\prime}, l_{i}^{\prime \prime}\right)$, $i=1,2, \ldots, k$. Оно состоит в том, чтобы распределения векторов $\eta_{i}=$ $x_{i} l_{i}^{\prime}+y_{i} l_{i}^{\prime \prime}$ были по возможности близки к вырожденным, что делает эти векторы более определенными, из-за чего, в частности, $\operatorname{Im} l_{i}^{\prime} \subseteq \operatorname{Im} l_{i}^{\prime \prime}$ и, как следствие,

$$
V_{s}=\sum_{i=1}^{k} \operatorname{Im} l_{i}^{\prime \prime}=\left\langle\operatorname{Im} l_{1}^{\prime \prime}, \operatorname{Im} l_{2}^{\prime \prime}, \ldots, \operatorname{Im} l_{k}^{\prime \prime}\right\rangle
$$

Как и для вектора $\eta$, мерой неопределенности каждого случайного вектора $\eta_{i} \in V_{s}, i=1,2, \ldots, k$, является энтропия $H\left(\eta_{i}\right)$ его распределения на $V_{s}$. Распределение $\eta_{i}$ вычисляется в предположении, что $x_{i} \in V_{n_{i}}$ выбирается случайно и равновероятно. Последнее предположение делается вопреки тому, что при фиксированном ключе $z \in V_{K}$ и равновероятном $a \in V_{N}$ распределение вектора $x_{i} \in V_{n_{i}}, i \in\{1, \ldots, k\}$, выражающегося в конечном счете через $a$ и $z$, вообще говоря, не обязано быть равномерным на $V_{n_{i}}$.

Второе требование заключается в согласованности набора линейных отображений $\mathfrak{L}=\left(\left(l_{i}^{\prime}, l_{i}^{\prime \prime}\right), i=1,2, \ldots, k\right)$ в том смысле, что сумма

$$
\eta_{\mathfrak{L}}=\sum_{i=1}^{k} \eta_{i}=\sum_{i=1}^{k}\left(x_{i} l_{i}^{\prime}+y_{i} l_{i}^{\prime \prime}\right)
$$

за счет использования выражений (3), (4) (но без использования выражений $\left.y_{i}=f_{i}\left(x_{i}\right), i=1,2, \ldots, k\right)$ должна приводиться к виду

$$
\eta=a L^{\prime}+z L+b L^{\prime \prime}
$$

для некоторых линейных отображений

$$
L^{\prime}: V_{N} \rightarrow V_{s}, \quad L: V_{K} \rightarrow V_{s}, \quad L^{\prime \prime}: V_{M} \rightarrow V_{s}
$$

Отображения

$$
L^{\prime}=L_{\mathfrak{L}}^{\prime}, \quad L=L_{\mathfrak{L}}, \quad L^{\prime \prime}=L_{\mathfrak{L}}^{\prime \prime}
$$

определяются по отображениям $l_{i}^{\prime}, l_{i}^{\prime \prime}, i=1,2, \ldots, k$, но сами промежуточные знаки шифрпреобразования $x_{i}, y_{i}$ должны сокращаться в сумме (10). 
Набор пар матриц $\mathfrak{L}=\left(\left(l_{i}^{\prime}, l_{i}^{\prime \prime}\right), i=1,2, \ldots, k\right)=\left(l^{\prime}, l^{\prime \prime}\right)$ порождает вектор $\eta_{\mathfrak{L}}$ вида (11), если найдется тройка матриц $\left(L^{\prime}, L, L^{\prime \prime}\right)$, для которой выполняется (с учетом (7)) цепочка тождеств

$$
\begin{aligned}
0 & =\eta+\eta_{\mathfrak{L}}=a L^{\prime}+z L+b L^{\prime \prime}+\sum_{i=1}^{k}\left(x_{i} l_{i}^{\prime}+y_{i} l_{i}^{\prime \prime}\right)= \\
& =a L^{\prime}+z L+b L^{\prime \prime}+x l^{\prime}+y l^{\prime \prime}=(a, y)\left(\begin{array}{c}
L^{\prime} \\
l^{\prime \prime}
\end{array}\right)+z L+(x, b)\left(\begin{array}{c}
l^{\prime} \\
L^{\prime \prime}
\end{array}\right)= \\
& =(a, y)\left(\begin{array}{c}
L^{\prime} \\
l^{\prime \prime}
\end{array}\right)+z L+\left((a, y) \widetilde{C}+z C_{0}\right)\left(\begin{array}{c}
l^{\prime} \\
L^{\prime \prime}
\end{array}\right)= \\
& =(a, y)\left[\left(\begin{array}{c}
L^{\prime} \\
l^{\prime \prime}
\end{array}\right)+\widetilde{C}\left(\begin{array}{c}
l^{\prime} \\
L^{\prime \prime}
\end{array}\right)\right]+z\left[L+C_{0}\left(\begin{array}{c}
l^{\prime} \\
L^{\prime \prime}
\end{array}\right)\right] .
\end{aligned}
$$

Определение. Набор матриц $\mathfrak{L}=\left(l^{\prime}, l^{\prime \prime}\right)$ назовем согласованным, если уравнение

$$
\widetilde{C}\left(\begin{array}{c}
l^{\prime} \\
L^{\prime \prime}
\end{array}\right)=\left(\begin{array}{l}
L^{\prime} \\
l^{\prime \prime}
\end{array}\right)
$$

разрешимо относительно неизвестных матриц $L^{\prime}, L^{\prime \prime}$ размеров, соответственно, $N \times s$ и $M \times s$.

В случае согласованности набора $\mathfrak{L}$ полагаем $L=C_{0}\left(\begin{array}{c}l^{\prime} \\ L^{\prime \prime}\end{array}\right)$.

Из равенства (12) и из первого требования к парам отображений $l_{i}^{\prime}, l_{i}^{\prime \prime}$, $i=1,2, \ldots, k$, следует, что $\operatorname{Im} L^{\prime} \subseteq \operatorname{Im} L^{\prime \prime}=V_{s}$.

Согласно определению множество $\mathfrak{W}_{s}$ всех согласованных наборов матриц $\mathfrak{L}=\left(l^{\prime}, l^{\prime \prime}\right)$ является векторным пространством над полем $G F(2)$. Если исключить из рассмотрения шифрпреобразования (1) с явными слабостями в виде тождественных равенств $a L^{\prime}=b L^{\prime \prime}+z L$, то уравнение $\widetilde{C}\left(\begin{array}{c}0 \\ L^{\prime \prime}\end{array}\right)=\left(\begin{array}{c}L^{\prime} \\ 0\end{array}\right)$ будет иметь только нулевое решение, что далее будем предполагать. Тогда элементы множества $\mathfrak{W}_{s}$ параметризуются парами матриц $l^{\prime}, L^{\prime \prime}$ (размеров, соответственно, $\sum_{i=1}^{k} n_{i} \times s$ и $\left.M \times s\right)$, которые могут быть произвольными. В результате получим равенство $\operatorname{dim}_{G F(2)} \mathfrak{W}_{s}=\left(M+\sum_{i=1}^{k} n_{i}\right) \cdot s$.

Для согласованной совокупности пар матриц $\mathfrak{L}=\left(l^{\prime}, l^{\prime \prime}\right)$ имеем цепочку равенств

$$
\eta_{\mathfrak{L}}=x l^{\prime}+y l^{\prime \prime}=\sum_{i=1}^{k}\left(x_{i} l_{i}^{\prime}+y_{i} l_{i}^{\prime \prime}\right)=\sum_{i=1}^{k} \eta_{i}=a L^{\prime}+z L+b L^{\prime \prime}=\eta .
$$


Если не заботиться о минимизации неопределенности случайного вектора $\eta \in V_{s}$ в выражении (13), то построение согласованных наборов пар матриц $\mathfrak{L}=\left(\left(l_{i}^{\prime}, l_{i}^{\prime \prime}\right), i=1,2, \ldots, k\right)$ не является проблемой. Опишем универсальный (но далеко не лучший) способ последовательного построения таких наборов $\mathfrak{L}$.

Для произвольной ненулевой матрицы $L^{\prime \prime}$ размера $M \times s$ по уравнению (12) однозначно восстанавливается матрица $l_{k}^{\prime \prime}$ размера $m_{k} \times s$. Матрица $l_{k}^{\prime}$ размера $n_{k} \times s$ выбирается затем исходя из минимизации энтропии $H\left(\eta_{k}\right)$ распределения случайного вектора $\eta_{k}$ в сумме (10). После этого аналогично строится матрица $l_{k-1}^{\prime}$ размера $n_{k-1} \times s$ при заданной матрице $l_{k-1}^{\prime \prime}$, однозначно восстанавливаемой по уравнению (12) и матрицам $L^{\prime \prime}, l_{k}^{\prime}$. Так можно поступать вплоть до построения матрицы $l_{1}^{\prime}$.

Проверку согласованности (12) можно упростить с помощью графического представления функциональной схемы, если строки матрицы $l_{i}^{\prime}$ приписывать в виде пометок к компонентам аргументов (к входам) отображения $f_{i}$ в (2), а строки матрицы $l_{i}^{\prime \prime}$ приписывать к компонентам значений $f_{i}$ (его выходам). Расстановка пометок (векторов из $V_{s}$ ) будет распространяться и на каждую линейную операцию в шифрпреобразовании (1). При этом, если $g: V_{n} \rightarrow V_{m}$, $V_{n} \ni x \mapsto x g=y \in V_{m},-$ одна из линейных операций в шифрпреобразовании, пометки входов $g$ образуют матрицу $\alpha^{\prime}$ размера $n \times s$, а пометки выходов $g$ - матрицу $\alpha^{\prime \prime}$ размера $m \times s$, то $\alpha^{\prime}=g \alpha^{\prime \prime}$. В частности, одинаковы пометки вокруг сумматора $\left(x_{1}, \ldots, x_{n}\right) \mapsto x_{1}+\ldots+x_{n}=y_{1}$; пометки вокруг узла размножения $x \mapsto(x, \ldots, x)=\left(y_{1}, \ldots, y_{m}\right)$ в сумме дают нулевой вектор из $V_{s}$; при $n=m$ и $g=\operatorname{id}_{V_{n}}$ обязательно $\alpha^{\prime}=\alpha^{\prime \prime}$. Последнее означает, что если какая-то компонента значения некоторого отображения в шифрпреобразовании (1) является компонентой аргумента другого какого-то одного отображения, то к этим компонентам приписываются одинаковые пометки.

\section{3. Восстановление элементов ключа}

Располагая согласованным набором матриц $\mathfrak{L}=\left(l^{\prime}, l^{\prime \prime}\right)$ и набором блоков открытого и шифрованного текстов $\left(a^{(j)}, b^{(j)}\right) \in V_{N} \times V_{M}, j=1,2, \ldots, T$, при заданном $z L$, как следует из равенства (13), можно получить $T$ реализаций случайного вектора $\eta$ в виде

$$
\sum_{i=1}^{k} \eta_{i}^{(j)}=\sum_{i=1}^{k}\left(x_{i}^{(j)} l_{i}^{\prime}+y_{i}^{(j)} l_{i}^{\prime \prime}\right)=a^{(j)} L^{\prime}+b^{(j)} L^{\prime \prime}+z L, \quad j=1,2, \ldots, T .
$$

Здесь $b^{(j)}=F\left(a^{(j)}, z\right)$, а $x_{i}^{(j)}$ и $y_{i}^{(j)}-$ аргументы и значения отображения $f_{i}, i=1,2, \ldots, k$, в случае, когда на вход всей функциональной схемы поступает вектор $a^{(j)}, \eta_{i}^{(j)}=x_{i}^{(j)} l_{i}^{\prime}+y_{i}^{(j)} l_{i}^{\prime \prime}$. При истинных $s$ ключевых битах $z L \in V_{s}$ совокупность векторов (14) должна в наибольшей степени отвечать распределению случайного вектора $\eta$. 
Обычно в ходе криптографического анализа в шифрпреобразование (1) не включается несколько начальных и/или конечных операций из всей их совокупности, предусмотренной алгоритмом шифрования. В этом случае векторы $a^{(j)}, b^{(j)}, j=1,2, \ldots, T$, не представляют открытый и шифрованный тексты, но они могут выражаться через открытый и шифрованный тексты с помощью ключевых элементов (не связанных, как правило, с $z L$ ), которые также могут отбраковываться в случае несоответствия набора векторов (14) гипотетическому распределению вектора $\eta$, правило вычисления которого описано ниже.

Статистическая отбраковка ключей по $T$ векторам (14) рассматривается в следующем разделе. Здесь только отметим, что эффективность отбраковки зависит, главным образом, от величины $\sigma=\sum_{v \in V_{s}} \varepsilon_{v}^{2}$, если распределение вектора $\eta \in V_{s}$ имеет вид $\left\{p_{v}=\frac{1}{2^{s}}+\varepsilon_{v}, v \in V_{s}\right\}$. Это не случайно, так как если $\left|\varepsilon_{v}\right| \ll \frac{1}{2^{s}}$ при всех $v \in V_{s}$, то для энтропии распределения вектора $\eta$ имеем

$$
H(\eta)=-\sum_{v \in V_{s}}\left(\frac{1}{2^{s}}+\varepsilon_{v}\right) \log _{2}\left(\frac{1}{2^{s}}+\varepsilon_{v}\right) \approx s-\frac{2^{s-1} \sigma}{\ln 2} .
$$

При оценивании распределения случайного вектора $\eta=\sum_{i=1}^{k} \eta_{i}$ предполагается, что случайные слагаемые $\eta_{i}$ независимы в совокупности, а $x_{i} \in V_{n_{i}}$, $i=1,2, \ldots, k$, равновероятны. Предположение о независимости, как уже отмечалось, приходится делать вопреки тому, что при заданном ключе $z \in V_{K}$ и случайном равновероятном входе $a \in V_{N}$ случайные векторы $x_{i} \in V_{n_{i}}$, $i=1,2, \ldots, k$, выражающиеся через $a$ и $z$, вообще говоря, являются зависимыми и не обязательно равновероятными. В результате при оценке необходимого числа $T$ блоков открытого и шифрованного текстов приходится вместо распределения $\left\{p_{v}=2^{-s}+\varepsilon_{v}, v \in V_{s}\right\}$ пользоваться другим распределением $\left\{\widetilde{p}_{v}=2^{-s}+\widetilde{\varepsilon}_{v}, v \in V_{s}\right\}$ и величиной $\widetilde{\sigma}=\sum_{v \in V_{s}} \widetilde{\varepsilon}_{v}^{2}$, вычисленными в предположении о независимости $\eta_{i}$ и равновероятности $x_{i} \in V_{n_{i}}, i=1,2, \ldots, k$. Заметим еще, что распределение $\left\{\widetilde{p}_{v}, v \in V_{s}\right\}$ зависит только от $s$ ключевых бит $z L \in V_{s}$, тогда как распределение $\left\{p_{v}, v \in V_{s}\right\}$ зависит, вообще говоря, от $K$ ключевых бит $z \in V_{K}$.

Эффективность многомерного линейного метода зависит от выбора согласованного набора пар матриц $\mathfrak{L}=\left(\left(l_{i}^{\prime}, l_{i}^{\prime \prime}\right), i=1,2, \ldots, k\right)$. Чем меньше энтропия $H(\eta)$ (или чем больше $\sigma$ ), тем меньшее количество материала $T$ потребуется для отбраковки ложных ключей. В этой связи являются желательными номера $i \in\{1, \ldots, k\}$, для которых $x_{i} l_{i}^{\prime}=f_{i}\left(x_{i}\right) l_{i}^{\prime \prime}$ при всех $x_{i} \in V_{n_{i}}$, в частности для которых $l_{i}^{\prime}=0, l_{i}^{\prime \prime}=0$. Такие $i$ не вносят неопределенности в случайный вектор $\eta$, поскольку $\eta_{i} \equiv 0$. В этой связи предпочтительнее согласованные наборы $\mathfrak{L}$ с минимально возможным показателем $\theta_{\mathfrak{L}}=\left|\left\{i \in\{1, \ldots, k\} \mid l_{i}^{\prime \prime} \neq 0\right\}\right|$. 
Однако следует иметь в виду, что если при построении набора $\mathfrak{L}=\left(\left(l_{i}^{\prime}, l_{i}^{\prime \prime}\right), i=1,2, \ldots, k\right)$ увеличивается число нулевых пар $\left(l_{i}^{\prime}, l_{i}^{\prime \prime}\right)$, $i \in\{1,2, \ldots, k\}$, то равенствами (12) накладываются более жесткие ограничения на компоненты остальных пар $\left(l_{i}^{\prime}, l_{i}^{\prime \prime}\right)$, уменьшающие свободу их выбора. Это может привести к завышению неопределенностей соответствующих ненулевых слагаемых $\eta_{i}$ в (10), а значит, и к увеличению неопределенности всей суммы $\eta$. Заметим еще, что для некоторого $i \in\{1,2, \ldots, k\}$ обязательно $l_{i}^{\prime \prime} \neq 0$. Иначе в случае $\widetilde{\sigma} \neq 0$ имели бы $l_{i}^{\prime}=0$ для всех $i=1,2, \ldots, k$. Тогда равенства (13) дают тождество $0=a L^{\prime}+z L+b L^{\prime \prime}$. Шифры с такой слабостью (при $L^{\prime \prime} \neq 0$ ) мы исключили из рассмотрения еще в предыдущем пункте (сразу после определения).

Распределение $\eta$ (при сделанных выше предположениях) оценивается сверткой

$$
\tilde{p}_{v}=\sum_{v_{1}+\ldots+v_{k}=v} p_{v_{1}}^{(1)} \cdot \ldots \cdot p_{v_{k}}^{(k)}, \quad v \in V_{s}
$$

распределений $\left\{p_{v}^{(i)}, v \in V_{s}\right\}$ отдельных слагаемых $\eta_{i}, i=1,2, \ldots, k$. Можно также предварительно перейти к величинам $c_{u}^{(i)}=\sum_{v \in V_{s}}(-1)^{(u, v)} p_{v}^{(i)}$, где $(u, v)=u_{1} v_{1}+\ldots+u_{s} v_{s},(-1)^{0}=1,(-1)^{1}=-1$. Для таких величин

$$
\begin{aligned}
p_{v}^{(i)} & =\frac{1}{2^{s}} \sum_{u \in V_{s}}(-1)^{(v, u)} c_{u}^{(i)}, \\
\widetilde{p}_{v} & =\frac{1}{2^{s}} \sum_{u \in V_{s}}(-1)^{(v, u)} c_{u}, \\
c_{u} & =c_{u}^{(1)} \cdot \ldots \cdot c_{u}^{(k)}=\sum_{v \in V_{s}}(-1)^{(u, v)} \widetilde{p}_{v},
\end{aligned}
$$

Наиболее приемлемым является экспериментальный способ оценки распределения случайного вектора $\eta$. Если вычислительные возможности не позволяют этого сделать, то можно прибегнуть к равенству $\eta=\eta_{1}+\ldots+\eta_{k}=$ $\left(\eta_{1}+\ldots+\eta_{i_{1}}\right)+\left(\eta_{i_{1}+1}+\ldots+\eta_{i_{2}}\right)+\ldots+\left(\eta_{i_{r-1}+1}+\ldots+\eta_{k}\right)$, в котором каждое слагаемое $\zeta_{j}=\eta_{i_{j-1}+1}+\ldots+\eta_{i_{j}}, j=1,2, \ldots, r, i_{r}=k$, представляется в виде (11) (со своими $a$ и $b$, состоящими из промежуточных знаков шифрпреобразования (1)), а распределение каждого случайного вектора $\zeta_{j}$ оценивается экспериментально. После этого распределение $\eta$ оценивается сверткой полученных распределений вероятностей случайных векторов $\zeta_{1}, \ldots, \zeta_{r}$. Чем меньше $r$, тем точнее будут получаемые приближения к распределению $\eta$. Но в любом случае часть зависимостей между $\eta_{1}, \ldots, \eta_{k}$ при таком подходе будет учтена. 


\section{2. Связь $s$-мерного и одномерного линейных методов}

\section{1}

Пусть для шифрпреобразования (1) построено $s$-мерное линейное вероятностное соотношение (8), в котором случайный вектор $\eta \in V_{s}$ имеет распределение

$$
\left\{p_{v}=\frac{1}{2^{s}}+\varepsilon_{v}, v \in V_{s}\right\}, \quad \sigma=\sum_{v \in V_{s}} \varepsilon_{v}^{2},
$$

а для вычисления векторов $a L^{\prime}$ и $b L^{\prime \prime}$ требуются, соответственно, ключевые элементы $z^{\prime}$ и $z^{\prime \prime}$. И пусть имеются открытый текст и соответствующий ему шифрованный текст, которые с помощью $z^{\prime}$ и $z^{\prime \prime}$ позволяют вычислить пары $\left(a^{(j)}, b^{(j)}\right) \in V_{N} \times V_{M}, b^{(j)}=F\left(a^{(j)}, z\right), j=1,2, \ldots, T$. Здесь $z \in V_{K}-$ ключевой вектор, участвующий (в отличие от $z^{\prime}$ и $z^{\prime \prime}$ ) в шифрпреобразовании (1).

После опробования $\left(z^{\prime}, z L, z^{\prime \prime}\right)$ (тройки ключевых элементов, в которой какие-то из трех компонент могут отсутствовать) получаем $T$ реализаций случайного вектора $\eta: \eta^{(j)}=a^{(j)} L^{\prime}+z L+b^{(j)} L^{\prime \prime}$. Ключевую пару $\left(z^{\prime}, z^{\prime \prime}\right)$ будем отбраковывать по статистике

$$
\xi=\sum_{v \in V_{s}} \frac{\left(\xi_{v}-T / S\right)^{2}}{T / S}, \quad \xi_{v}=\left|\left\{j \in\{1,2, \ldots, T\} \mid \eta^{(j)}=v\right\}\right|, \quad S=2^{s} .
$$

Это одна из возможных статистик для отбраковки $\left(z^{\prime}, z^{\prime \prime}\right)$. Статистика $\xi$ не зависит от $z L$, поэтому при вычислении частот $\xi_{v}, v \in V_{s}$, можно полагать $z L=0$, а опробовать только $\left(z^{\prime}, z^{\prime \prime}\right)$. Элементы ключа $z L$ обычно потом доопробуются. Они могут также восстанавливаться по статистике

$$
\xi^{\prime}=\sum_{v \in V_{s}} \frac{\left(\xi_{v}-T \widetilde{p}_{v}\right)^{2}}{T \widetilde{p}_{v}}
$$

в которой частоты $\xi_{v}, v \in V_{s}$, вычисляются (при известных $\left(z^{\prime}, z^{\prime \prime}\right)$ ) для опробуемых $z L \in V_{s}$. Эффективность статистики $\xi^{\prime}$ в настоящей работе не оценивается.

В случае ложной пары $\left(z^{\prime}, z^{\prime \prime}\right)$ (гипотеза $H_{1}$ ) полагаем $\mathbf{P}_{1}\{\eta=v\}=\frac{1}{S}$ для всех $v \in V_{s}$. Тогда

$$
\mathbf{M}_{1} \xi=S-1, \quad \mathbf{D}_{1} \xi=2(S-1)-\frac{2(S-1)}{T} .
$$


При больших $T$, как видим, $\mathbf{D}_{1} \xi \approx 2(S-1)$, а распределение статистики $\xi$ хорошо аппроксимируется распределением $\chi_{m}^{2}$ с плотностью

$$
\rho(x)=\frac{1}{\Gamma(m / 2) 2^{m / 2}} x^{m / 2-1} e^{-x / 2}
$$

где $m=S-1$ есть число степеней свободы [14]. С помощью этого распределения можно оценивать величину $C$, для которой $\mathbf{P}_{1}\{\xi \geqslant C\} \leqslant \beta, \beta$ - заранее задаваемая верхняя оценка вероятности ошибки второго рода, т. е. вероятности принять ложные ключевые тройки за истинные.

В случае истинной пары $\left(z^{\prime}, z^{\prime \prime}\right)$ (гипотеза $\left.H_{0}\right)$ полагаем

$$
\mathbf{P}_{0}\{\eta=v\}=\frac{1}{S}+\varepsilon_{v}, \quad v \in V_{s} .
$$

Тогда (см. [15])

$$
\begin{aligned}
& \mathbf{M}_{0} \xi=(T-1) S \sigma+S-1 \\
& \mathbf{D}_{0} \xi=\frac{2 S^{2}(T-1)}{T}\left\{\left(1-\frac{1}{S}-\sigma\right)\left(\frac{1}{S}+\sigma\right)+2(T-2)\left[\frac{\sigma}{S}-\sum_{v \in V_{s}} \varepsilon_{v}^{3}-\sigma^{2}\right]\right\} .
\end{aligned}
$$

При больших $T$ и малых $\sigma$, как видим, $\mathbf{D}_{0} \xi \approx 2(2(T-1) S \sigma+S-1)$, а распределение статистики $\xi$ хорошо аппроксимируется нецентральным распределением $\chi_{m}^{2}(\lambda)$ с параметром нецентральности $\lambda=(T-1) S \sigma$ и с плотностью

$$
h(x)=\frac{1}{\Gamma(1 / 2) 2^{m / 2}} x^{m / 2-1} e^{-(x+\lambda) / 2} \sum_{j=0}^{\infty} \frac{\lambda^{j} x^{j}}{(2 j) !} \frac{\Gamma(1 / 2+j)}{\Gamma(m / 2+j}, \quad m=S-1 .
$$

Среднее и дисперсия этого распределения равны соответственно $\lambda+m$ и $2(2 \lambda+m)$.

Для оценки вероятности ошибки первого рода $\alpha=\mathbf{P}_{0}\{\xi<C\}-$ вероятности принять истинные $\left(z^{\prime}, z^{\prime \prime}\right)$ за ложные - лучше пользоваться приближениями плотности $h(x)$. В [16] предлагается для соответствующих случайных величин использовать замену

$$
\chi^{\prime}=\frac{m+\lambda}{m+2 \lambda} \chi_{m}^{2}(\lambda) \quad \text { c } \quad \mathbf{M} \chi^{\prime}=g=\frac{(m+\lambda)^{2}}{m+2 \lambda}
$$

полезную тем, что плотность $\chi^{\prime}$ хорошо аппроксимируется плотностью $\chi_{g}^{2}$. 
В [17] эта замена уточняется: предлагается рассматривать

$$
\chi^{\prime \prime}=\frac{m+2 \lambda}{m+3 \lambda}\left(\chi_{m}^{2}(\lambda)+\frac{\lambda^{2}}{m+3 \lambda}\right) \quad \text { с } \quad \mathbf{M} \chi^{\prime \prime}=f=\frac{(m+2 \lambda)^{3}}{(m+3 \lambda)^{2}} .
$$

Среднее, дисперсия и центральный третий момент для $\chi^{\prime \prime}$ соотносятся как $1: 2: 8$ (как и в случае $\chi_{f}^{2}$ ). Плотность распределения $\chi^{\prime \prime}$ более точно аппроксимируется плотностью $\chi_{f}^{2}$. Здесь числа степеней свободы $g$ и $f$ не обязательно целые. Плотности распределений $\chi_{g}^{2}$ и $\chi_{f}^{2}$ представляются формулой (15), если в ней заменять $m$ соответственно на $g$ и $f$.

При оценке величины необходимого материала $T$ во всех вычислениях будем ограничиваться главными членами в асимптотических разложениях, предполагая $\beta \rightarrow 0,0<\alpha \leqslant \alpha_{0}<1, \sigma \rightarrow 0$. Целое $s>0$ и вещественное $\alpha_{0} \in(0,1)$ считаем фиксированными. Тогда $C \rightarrow \infty, \mathbf{M}_{0} \xi \rightarrow \infty$, $(T-1) \sigma \rightarrow \infty$ и поэтому $T \rightarrow \infty$. В этих предположениях использование для наших целей распределения $\chi^{\prime \prime}$ и плотности $p_{1}(x)$ правомерно.

Нижнюю оценку для $C>m$ получаем из следующей цепочки неравенств

$$
\begin{aligned}
\beta & \geqslant \mathbf{P}_{1}\{\xi \geqslant C\} \simeq \int_{C}^{+\infty} \rho(x) d x=\frac{1}{\Gamma(m / 2) 2^{m / 2}} \int_{C}^{+\infty} x^{m / 2-1} e^{-x / 2} d x> \\
& >\frac{C^{m / 2-1}}{\Gamma(m / 2) 2^{m / 2}} \int_{C}^{+\infty} e^{-x / 2} d x=\frac{1}{\Gamma(m / 2)}\left(\frac{C}{2}\right)^{m / 2-1} e^{-C / 2}>e^{-C / 2}
\end{aligned}
$$

После логарифмирования крайних значений в данной цепочке получим

$$
C \gtrsim 2 \ln \beta^{-1}
$$

Нижней оценкой для $C$ выступает величина, эквивалентная $2 \ln \beta^{-1}$ при $T \rightarrow \infty, \beta \rightarrow 0$.

В конкретных условиях вместо асимптотических оценок можно воспользоваться оценками из [18]. Так, при $m \geqslant 2$ и $\beta \leqslant 0.17$ по предложению 5.1 в [18] имеем $C \geqslant 2 \ln \beta^{-1}+m-5 / 2$. При других ограничениях на $m$ и $\beta$ теоремы 5.1 и 5.2 в [18] дают еще более точные оценки для $C$ с главным членом $2 \ln \beta^{-1}$. 
Для оценки необходимой величины материала $T$ воспользуемся неравенством

$$
C \lesssim \lambda=(T-1) S \sigma=\mathbf{M}_{0} \xi-\mathbf{M}_{1} \xi .
$$

Это неравенство очевидно в случае $\mathbf{M}_{1} \xi \leqslant C \leqslant \mathbf{M}_{0} \xi$. Пусть $C>\mathbf{M}_{0} \xi$ и $\Delta=C-\mathbf{M}_{0} \xi$. Тогда в силу неравенства Чебышева

$$
\begin{aligned}
1-\alpha_{0} & \leqslant 1-\alpha=\mathbf{P}_{0}\{\xi \geqslant C\} \leqslant \mathbf{P}_{0}\left\{\left|\xi-\mathbf{M}_{0} \xi\right| \geqslant \Delta\right\} \leqslant \\
& \leqslant \frac{2(S-1)+4 \lambda}{\Delta^{2}} .
\end{aligned}
$$

Сравнивая крайние члены в этой цепочке неравенств, получаем

$$
C \leqslant(S-1)+\lambda+\sqrt{\frac{2(S-1)+4 \lambda}{1-\alpha_{0}}},
$$

поэтому (в наших условиях)

$$
C \lesssim \lambda+\frac{2}{\sqrt{1-\alpha_{0}}} \sqrt{\lambda} \lesssim \lambda, \quad \lambda \rightarrow \infty
$$

Доказанное в результате неравенство (17) вместе с (16) в условиях наших предположений для $T=\frac{\lambda}{S \sigma}+1$ дает неравенство

$$
T \gtrsim \frac{2 \ln \beta^{-1}}{S \sigma} .
$$

Для получения более точных оценок величины $T$ можно обратиться к распределению $\chi^{\prime \prime}$, переходя к статистике

$$
\xi^{\prime \prime}=\frac{m+2 \lambda}{m+3 \lambda}\left(\xi+\frac{\lambda^{2}}{m+3 \lambda}\right)
$$

и используя для верхней оценки величины

$$
\frac{m+2 \lambda}{m+3 \lambda}\left(C+\frac{\lambda^{2}}{m+3 \lambda}\right)
$$

теорему А или одну из теорем 4.1 и 4.2 из [18], в которых в качестве числа степеней свободы $k$ следует использовать

$$
f=\frac{(m+2 \lambda)^{3}}{(m+3 \lambda)^{2}} .
$$

2017, T. 8, № 4, С. 29-62 


\section{2}

В настоящем пункте рассматривается одномерный линейный метод, использующий для отбраковки ключей несколько вероятностных линейных соотношений, полученных из одного многомерного вероятностного линейного соотношения.

Пусть для шифрпреобразования (1) построено $s$-мерное вероятностное линейное соотношение (8), в котором случайный вектор $\eta=a L^{\prime}+z L+b L^{\prime \prime} \in V_{s}$ имеет распределение $\left\{p_{v}=2^{-s}+\varepsilon_{v}, v \in V_{s}\right\}$. Обозначим через $V_{s}^{*}$ множество двоичных вектор-столбцов размера $s$. При каждом ненулевом вектор-столбце $l \in V_{s}^{*} \backslash\{0\}$, полагая $l^{\prime}=L^{\prime} l, \widehat{l}=L l, l^{\prime \prime}=L^{\prime \prime} l$, из (8) получаем одномерное линейное вероятностное соотношение

$$
b l^{\prime \prime}=a l^{\prime}+z \widehat{l}+\eta l,
$$

в котором $l^{\prime \prime} \in V_{M}^{*}, l^{\prime} \in V_{N}^{*}, \widehat{l} \in V_{K}^{*}, \eta l \in G F(2)-$ случайный бит,

$$
\mathbf{P}\{\eta l=0\}=\frac{1}{2}+\sum_{v \in V_{s}: v l=0} \varepsilon_{v}, \quad \mathbf{P}\{\eta l=1\}=\frac{1}{2}+\sum_{v \in V_{s}: v l=1} \varepsilon_{v} .
$$

Обозначим

$$
\triangle_{l}=\sum_{v \in V_{s}: v l=0} \varepsilon_{v}, \quad \delta_{l}=2 \triangle_{l}
$$

Так как $\sum_{v \in V_{s}} \varepsilon_{v}=0$, то $\sum_{v \in V_{s}: v l=1} \varepsilon_{v}=-\triangle_{l}$, и

$$
\mathbf{P}\{\eta l=0\}=\frac{1+\delta_{l}}{2}, \quad \mathbf{P}\{\eta l=1\}=\frac{1-\delta_{l}}{2} .
$$

Энтропия случайного вектора $\eta$ имеет вид

$$
s-\frac{2^{s-1} \sigma}{\ln 2}+o(\sigma), \quad \sigma=\sum_{v \in V_{s}} \varepsilon_{v}^{2} .
$$

Аналогично, энтропия случайного бита $\eta l$ имеет вид $1-\delta_{l}^{2} /(2 \ln 2)+o\left(\delta_{l}^{2}\right)$.

Вместо случайного вектора $\eta$ далее используются $s$ его координат в специально подбираемом базисе, а именно случайные биты $\eta_{i}, i=1,2, \ldots, s$, где вектор-столбцы $l_{1}, \ldots, l_{s} \in V_{s}^{*} \backslash\{0\}$ линейно независимы. Ориентируясь на максимальные значения $\left|\delta_{l_{i}}\right|$, можно добиться, по крайней мере, выполнения неравенства

$$
\delta_{l_{1}}^{2}+\ldots+\delta_{l_{s}}^{2} \geqslant \frac{2^{s} \sigma}{\left\lceil\frac{2^{s}-1}{s}\right\rceil} \simeq s \sigma,
$$

которое обосновывается следующей теоремой. 
Теорема 1. Справедливо равенство

$$
\sum_{l \in V_{s}^{*} \backslash\{0\}} \delta_{l}^{2}=2^{s} \sigma .
$$

Доказательство. Так как $\sum_{v \in V_{s}} \varepsilon_{v}=0$, то

$0=\left(\sum_{v \in V_{s}} \varepsilon_{v}\right)^{2}=\sum_{v \in V_{s}} \varepsilon_{v}^{2}+2 \sum_{v \neq w} \varepsilon_{v} \varepsilon_{w}=\sigma+\Sigma, \quad$ где $\quad \Sigma=2 \sum_{v \neq w} \varepsilon_{v} \varepsilon_{w}=-\sigma$.

(Здесь и далее в доказательстве при суммировании по неупорядоченным подмножествам мощности 2 из $V_{s}$ область суммирования под знаком суммы обозначается формулой $v \neq w$.) Справедливо также равенство

$$
\begin{aligned}
2 \sum_{l \in V_{s}^{*} \backslash\{0\}} \triangle_{l}^{2} & =\sum_{l \in V_{s}^{*} \backslash\{0\}}\left[\triangle_{l}^{2}+\left(-\triangle_{l}\right)^{2}\right]= \\
& =\sum_{l \in V_{s}^{*} \backslash\{0\}}\left[\left(\sum_{v \in V_{s}: v l=0} \varepsilon_{v}\right)^{2}+\left(\sum_{v \in V_{s}: v l=1} \varepsilon_{v}\right)^{2}\right]= \\
& =\left(2^{s}-1\right) \sigma+\sum_{l \in V_{s}^{*} \backslash\{0\}}\left[2 \sum_{\substack{v \neq w: \\
v l=w l=0}} \varepsilon_{v} \varepsilon_{w}+2 \sum_{\substack{v \neq w: \\
v l=w l=1}} \varepsilon_{v} \varepsilon_{w}\right]= \\
& =\left(2^{s}-1\right) \sigma+\left(2^{s-1}-1\right) \Sigma=\left(2^{s}-1\right) \sigma-\left(2^{s-1}-1\right) \sigma= \\
& =2^{s-1} \sigma .
\end{aligned}
$$

В последних переходах использовалось то обстоятельство, что каждая пара различных векторов $v, w$ содержится ровно в $2^{s-1}-1$ гиперплоскостях пространства $V_{s}$ (из $2^{s+1}-2$ возможных) по числу линейных гиперплоскостей в фактор-пространстве $V_{s} /<v+w>\cong V_{s-1}$. Из полученного тождества

$$
2 \sum_{l \in V_{s}^{*} \backslash\{0\}} \triangle_{l}^{2}=2^{s-1} \sigma
$$

и равенства $4 \triangle_{l}^{2}=\delta_{l}^{2}, l \in V_{s}^{*} \backslash\{0\}$, следует (20). Теорема 1 доказана.

Справедливость теоремы следует также из равенства Парсеваля, относящегося к преобразованию Фурье, рассмотренному в предыдущем разделе в П. 1.3. 
Следствие. Можно так выбрать систему линейно независимых векторстолбиов $l_{1}, \ldots, l_{s} \in V_{s}^{*} \backslash\{0\}$, что

$$
\delta_{l_{1}}^{2}+\ldots+\delta_{l_{s}}^{2} \geqslant \frac{2^{s} \sigma}{æ_{s}}, \quad \text { где } æ_{s}=\left\lceil\frac{2^{s}-1}{s}\right\rceil .
$$

Доказательство данного утверждения следует из того, что ненулевые векторы $s$-мерного линейного пространства над полем $G F(2)$ общим числом $2^{s}-1=r \cdot s+a, a<s$, могут быть разбиты на $r$ непересекающихся подмножеств мощности и ранга $s$ и еще одного подмножества мощности и ранга $a$. Наличие такого разбиения обеспечивает, например, последовательность $1, \vartheta, \vartheta^{2}, \ldots, \vartheta^{i}, \vartheta^{i+1}, \ldots, \vartheta^{2^{s}-2}$ ненулевых элементов поля $G F\left(2^{s}\right)$ с примитивным элементом $\vartheta$. В этой последовательности элементы $\vartheta^{i}, \vartheta^{i+1}, \ldots, \vartheta^{i+s-1}$ линейно независимы над $G F(2)$ для всех $i=0,1, \ldots, 2^{s}-s-1$. Следствие доказано.

Пусть $l_{1}, \ldots, l_{s} \in V_{s}^{*} \backslash\{0\}$ - система линейно независимых векторстолбцов. Обозначим $\delta_{i}=\delta_{l_{i}}, i=1,2, \ldots, s$. Как и в предыдущем пункте, будем считать, что $\beta \rightarrow 0, \sigma \rightarrow 0, T \rightarrow \infty, 0<\alpha \leqslant \alpha_{0}<1$, где $\alpha_{0} \in(0,1)$ и $s \in \mathbb{N}$ фиксированы.

Для отбраковки ключевых троек $\left(z^{\prime}, z L, z^{\prime \prime}\right)$ вместо статистики $\xi$, рассмотренной в предыдущем пункте, обратимся к статистике

$$
\zeta=\delta_{1} \zeta_{1}+\ldots+\delta_{s} \zeta_{s}-\frac{T}{2} \sum_{i=1}^{s} \delta_{i}
$$

где $\zeta_{i}=\left|\left\{j \in\{1,2, \ldots, T\} \mid \eta^{(j)} l_{i}=0\right\}\right|, i=1,2, \ldots, s$. Здесь, напомним, $\eta^{(j)}=a^{(j)} L^{\prime}+z L+b^{(j)} L^{\prime \prime}$. Для отбраковки ключевой тройки $\left(z^{\prime}, z L, z^{\prime \prime}\right)$ нужно различать прежние две гипотезы $H_{0}$ и $H_{1}$, при которых

$\mathbf{M}_{1} \zeta=0, \quad \mathbf{D}_{1} \zeta=\frac{T}{4} \sigma_{s}, \quad \mathbf{M}_{0} \zeta=\frac{T}{2} \sigma_{s}, \quad \mathbf{D}_{0} \zeta \simeq \frac{T}{4} \sigma_{s}, \quad \sigma_{s}=\delta_{1}^{2}+\ldots+\delta_{s}^{2}$.

В обоих случаях распределение $\zeta$ хорошо приближается нормальным распределением. Величина необходимого материала $T$ оценивается по той же схеме, что и в предыдущем пункте, с помощью приближения

$$
\frac{1}{\sqrt{2 \pi}} \int_{t}^{+\infty} e^{-x^{2} / 2} d x \approx \frac{1}{\sqrt{2 \pi}} \frac{e^{-t^{2} / 2}}{t}
$$

для больших $t[19]$. 
При отбраковке по статистике $\zeta / \sqrt{T \sigma_{s} / 4}$ аналогом неравенства (16) будет $C \gtrsim \sqrt{2 \ln \beta^{-1}}$, а аналогом неравенства (18) будет неравенство

$$
T \gtrsim \frac{2 \ln \beta^{-1}}{\sigma_{s}}
$$

Из теоремы 1 следует, что $\sigma_{s} \leqslant S \sigma$, поэтому нижняя оценка в (21) заведомо больше, чем в (18), но не больше, чем в $2^{s} / s$ раз. В то же время не следует исключать ситуации, когда $\sigma_{s}$ незначительно отличается от $2^{s} \sigma$. Тогда преимущество многомерного линейного метода может нивелироваться большей трудоемкостью вычисления статистики $\xi$ по сравнению с трудоемкостью вычисления статистики $\zeta$.

Подчеркнем, что данные качественные рассуждения не могут обосновывать преимущество одного из этих методов хотя бы потому, что при получении оценок (18) и (21) не привлекались наиболее мощные статистические критерии. Рассчитывать же эффективность наиболее мощных статистических критериев на основе величин $\widetilde{\varepsilon}_{v}, v \in V_{s}$, представляется нецелесообразным, поскольку нет точных оценок близости $\varepsilon_{v}$ и $\widetilde{\varepsilon}_{v}, v \in V_{s}$. Ответ же на вопрос о преимуществе многомерного линейного метода, скорей всего, зависит от конкретного шифрпреобразования и условий проведения криптографических анализов.

\section{3}

Если соотношение (8) получается в результате суммирования соотношений (9), то соотношение (19) представляет собой сумму соотношений $y_{i} l_{i}^{\prime \prime} l=x_{i} l_{i}^{\prime} l+\eta_{i} l, i=1,2, \ldots, k$, получающихся из равенств (9) умножением справа на $l \in V_{s}^{*} \backslash\{0\}$. Выше вместо статистики $\xi$ использовалась статистика $\zeta$, построенная по $l$ вектор-столбцам $l_{1}, \ldots, l_{s}$. Обратно, при наличии $s>1$ вероятностных одномерных линейных соотношений вместо статистики $\zeta$ из п. 2.2 можно использовать статистику $\xi$ из п. 2.1 , относящуюся к $s$-мерному линейному методу.

Действительно, пусть одномерные вероятностные линейные соотношения $b l^{\prime \prime(j)}=a l^{(j)}+z \widehat{l}^{(j)}+\eta^{(j)}, l^{\prime \prime(j)} \in V_{M}^{*}, l^{\prime(j)} \in V_{N}^{*}, \widehat{l}^{(j)} \in V_{K}^{*}$, определяются по согласованным наборам вектор-столбцов $\mathfrak{L}^{(j)}=\left(\left(l_{i}^{(j)}, l_{i}^{\prime \prime(j)}\right), i=1,2, \ldots, k\right) \in \mathfrak{M}_{1}$, $j=1,2, \ldots, s$. Последние определяют согласованный набор матриц $\mathfrak{L}=\left(\left(l_{i}^{\prime}, l_{i}^{\prime \prime}\right), i=1,2, \ldots, k\right) \in \mathfrak{M}_{s}$. Матрицы $l_{i}^{\prime}$ и $l_{i}^{\prime \prime}$ состоят, соответственно, из столбцов $l_{i}^{\prime(j)}$ и $l_{i}^{\prime \prime(j)}, j=1,2, \ldots, s$. Статистика $\xi$ соответствует $s$-мерному вероятностному линейному соотношению (8), отвечающему набору $\mathfrak{L}$. Матрицы $L^{\prime \prime}, L^{\prime}, L$ в (8) составлены, соответственно, из столбцов $l^{\prime \prime(j)}, l^{\prime(j)}, \widehat{l}^{(j)}, j=1,2, \ldots, s$, a $\eta=\left(\eta^{(1)}, \ldots, \eta^{(s)}\right)$. 


\section{3. Показатели рассеивания линейной среды шифрпреобразований и невырожденных матриц}

Линейный метод для $s=1$ (как уже отмечалось во введении) показал, что степень рассеивания шифрпреобразования разумно измерять специальной численной характеристикой, названной показателем рассеивания линейной среды шифрпреобразования. В дальнейшем это привело к появлению новых классов алгоритмов блочного шифрования (XSL-схемы, вложенные схемы Фейстеля). В настоящем разделе приводятся уточнения оценок показателей рассеивания, связанные с многомерным линейным методом (когда $s>1$ ).

3.1

В случае $s=1$ показатель рассеивания линейной среды $C$ шифрпреобразования (1) определяется (см. [13]) формулой

$$
\theta_{1}(C)=\min _{\mathfrak{L} \in \mathfrak{W}_{1} \backslash\{0\}} \theta_{\mathfrak{L}},
$$

где, напомним, $\theta_{\mathfrak{L}}=\left|\left\{i \in\{1,2, \ldots, k\} \mid l_{i}^{\prime \prime} \neq 0\right\}\right|$. Если $\mathfrak{L} \in \mathfrak{W}_{1}$ и $l_{i}^{\prime \prime}=0$, то $l_{i}^{\prime}=0$. Тогда $\eta_{i} \equiv 0$ имеет вырожденное распределение и $\eta_{i}$ не вносит неопределенности в сумму $\eta$.

При $s=1$ неопределенность $\eta_{i}, i=1,2, \ldots, k$, характеризуется преобладанием $\delta_{i}=2 \mathbf{P}\left\{\eta_{i}=0\right\}-1$, а неопределенность $\eta=\eta_{1}+\ldots+\eta_{k}$ оценивается величиной $\widetilde{\delta}=\widetilde{\delta}_{\mathfrak{L}}=\delta_{1} \cdot \ldots \cdot \delta_{k}$.

Важность показателя $\theta_{1}(C)$ определяется тем, что при наличии его нижней оценки $\theta \leqslant \theta_{1}(C)$, т. е. когда $\theta \leqslant \theta_{\mathfrak{L}}$ для любой системы $\mathfrak{L} \in \mathfrak{W}_{1} \backslash\{0\}$, можно гарантировать выполнение неравенства

$$
|\widetilde{\delta}| \leqslant \triangle^{\theta}
$$

если

$$
\max _{i=1,2, \ldots, k} \max _{\substack{l_{i}^{\prime} \in V_{n_{i}}^{*}, l_{i}^{\prime \prime} \in V_{m_{i}}^{*} \backslash\{0\}}}\left|\delta_{i}\right| \leqslant \triangle .
$$

Показатель $\theta_{1}(C)$ позволяет также ранжировать различные невырожденные линейные преобразования пространства $V_{n}=G F(2)^{n}$ по их криптографическим качествам. Конкретно, в настоящем пункте речь пойдет о матрицах $\Lambda \in G L(n, 2)$, используемых в так называемых канонических $X S L$-шифрах на $V_{n}, n=m \cdot \varkappa$, одна итерация которых осуществляет два преобразования. 
Первое $S$ - нелинейное,

$$
S=(\underbrace{\pi, \ldots, \pi}_{\varkappa}), \quad \pi \in S_{V_{m}} .
$$

Если $v=\left(v_{1}, \ldots, v_{\varkappa}\right) \in\left(V_{m}\right)^{\varkappa}=V_{n}$, то $S(v)=\left(\pi\left(v_{1}\right), \ldots, \pi\left(v_{\varkappa}\right)\right)$.

Второе преобразование осуществляет умножение вектор-строк из $V_{n}$ на матрицу $\Lambda$. Итерационные ключи в каноническом $X S L$-шифре считаем нулевыми, поэтому $X=\mathrm{id}_{V_{n}}$ не рассматриваем. Последнее предположение допустимо, поскольку при наложении итерационных ключей покомпонентно по $\bmod 2$ значения $\left|\delta_{i}\right|, i=1,2, \ldots, k$, и $|\widetilde{\delta}|$ не зависят от ключей.

Функциональная схема $t$ итераций канонического $X S L$-шифра (1) с $N=M=n$ состоит из $k=\varkappa t$ преобразований (2), в которых $n_{i}=m_{i}=m$, $f_{i}=\pi, i=1,2, \ldots, \varkappa t$. Линейная среда этого шифра представляется блочнодиагональной матрицей $C \in G L((t+1) n, 2)$ с $t$ блоками $\Lambda \in G L(n, 2)$. В левом верхнем углу матрицы $C$ располагается блок $I$ в виде единичной матрицы из $G L(n, 2)$. Если $x_{i} \in V_{n}$ - аргумент $S$ на $i$-й итерации, $i=1,2, \ldots, t$, а $y_{i}=S\left(x_{i}\right)$, то $\left(a, y_{1}, \ldots, y_{t}\right) C=\left(x_{1}, \ldots, x_{t}, b\right), b=F(a)$.

Для канонического $X S L$-шифра в случае линейного метода $(s=1)$ имеем

$$
\eta=\sum_{i=1}^{t}\left(x_{i} l_{i}^{\prime}+y_{i} l_{i}^{\prime \prime}\right)=\sum_{i=1}^{t} \sum_{j=1}^{\varkappa}\left(x_{i j} l_{i j}^{\prime}+y_{i j} l_{i j}^{\prime \prime}\right),
$$

где $x_{i}=\left(x_{i 1}, \ldots, x_{i \varkappa}\right), y_{i}=\left(y_{i 1}, \ldots, y_{i \varkappa}\right), x_{i j}, y_{i j} \in V_{m}$, вектор-столбцы $l_{i}^{\prime}, l_{i}^{\prime \prime} \in V_{n}^{*}$ состоят из вектор-столбцов $l_{i j}^{\prime}, l_{i j}^{\prime \prime} \in V_{m}^{*}$. Согласованность набора вектор-столбцов

$$
\mathfrak{L}=\left(\left(l_{i}^{\prime}, l_{i}^{\prime \prime}\right), i=1,2, \ldots, t\right)=\left(\left(l_{i j}^{\prime}, l_{i j}^{\prime \prime}\right), i=1,2, \ldots, t, j=1,2, \ldots, \varkappa\right)
$$

означает выполнение равенств $l_{i}^{\prime \prime}=\Lambda l_{i+1}^{\prime}, i=1,2, \ldots, t-1$. Тогда

$$
\eta=a l_{1}^{\prime}+b \Lambda^{-1} l_{t}^{\prime \prime}, \quad L^{\prime}=l_{1}^{\prime}, \quad L^{\prime \prime}=\Lambda^{-1} l_{t}^{\prime \prime} .
$$

При определении показателя рассеивания $\theta_{1}(C)$ по формуле (22) в случае канонического $X S L$-шифра следует $\mathfrak{W}_{1}$ заменить на

$$
\mathfrak{W}_{1}^{(0)}=\left\{\mathfrak{L} \in \mathfrak{W}_{1} \mid l_{i j}^{\prime}=0 \Leftrightarrow l_{i j}^{\prime \prime}=0, i=1,2, \ldots, t, j=1,2, \ldots, \varkappa\right\},
$$

так как при $l_{i j}^{\prime}=0$ и $l_{i j}^{\prime \prime} \neq 0$ случайный бит $\eta_{i j}=x_{i j} l_{i j}^{\prime}+y_{i j} l_{i j}^{\prime \prime}=\pi\left(x_{i j}\right) l_{i j}^{\prime \prime}$ равновероятен в силу биективности отображения $f_{(i-1) \varkappa+j}$, следовательно, $\delta_{(i-1) \varkappa+j}=0$ и потому $\widetilde{\delta}_{\mathfrak{L}}=0$. 
Если канонический $X S L$-шифр состоит из $t=2$ итераций, то, применяя формулу (22), получим характеристику для матрицы $\Lambda$. Тогда для $\mathfrak{L} \in \mathfrak{W}_{1}^{(0)}$ имеем $\theta_{\mathfrak{L}}=w\left(l_{1}^{\prime \prime}\right)+w\left(l_{2}^{\prime}\right)=w\left(l_{1}^{\prime}\right)+w\left(l_{2}^{\prime \prime}\right)$, если для вектор-столбца $l \in V_{n}^{*}$, составленного из вектор-столбцов $l_{1}, \ldots, l_{\varkappa} \in V_{m}^{*}$, полагать $w(l)=\mid\{j \in$ $\left.\{1, \ldots, \varkappa\} \mid l_{j} \neq 0\right\} \mid$. Правая часть формулы (22) в этом случае равна величине

$$
\min _{l_{2}^{\prime} \in V_{n}^{*} \backslash\{0\}}\left(w\left(l_{1}^{\prime \prime}\right)+w\left(l_{2}^{\prime}\right)\right)=\min _{l_{2}^{\prime} \in V_{n}^{*} \backslash\{0\}}\left(w\left(\Lambda l_{2}^{\prime}\right)+w\left(l_{2}^{\prime}\right)\right),
$$

характеризующей матрицу $\Lambda$. Обозначим ее через $\rho_{1,2}(\Lambda)$ :

$$
\rho_{1,2}(\Lambda)=\min _{l \in V_{n}^{*} \backslash\{0\}}(w(l)+w(\Lambda l)) .
$$

Индекс 1 в обозначении $\rho_{1,2}(\Lambda)$ соответствует $s=1$, а индекс 2 указывает число итераций $t=2$. Показатель $\rho_{1,2}(\Lambda)$ называется коэффициентом рассеивания матрицы $\Lambda \in G L(n, 2)$ относительно линейного метода при ее разбиении на $\varkappa$ полос по $m$ строк в полосе и на $\varkappa$ колонок по $m$ столбцов в колонке.

При числе итераций $t>2$ в каноническом $X S L$-шифре для произвольной согласованной системы вектор-столбцов $\mathfrak{L} \in \mathfrak{W}_{1}$ справедливы (см. [13]) соотношения

$$
\begin{aligned}
2 \theta_{\mathfrak{L}} & =w\left(l_{1}^{\prime}\right)+w\left(l_{1}^{\prime \prime}\right)+w\left(l_{2}^{\prime}\right)+w\left(l_{2}^{\prime \prime}\right)+\ldots+w\left(l_{t-1}^{\prime}\right)+w\left(l_{t-1}^{\prime \prime}\right)+w\left(l_{t}^{\prime}\right)+w\left(l_{t}^{\prime \prime}\right)= \\
& =w\left(l_{1}^{\prime}\right)+w\left(\Lambda l_{2}^{\prime}\right)+w\left(l_{2}^{\prime}\right)+w\left(\Lambda l_{3}^{\prime}\right)+\ldots+w\left(l_{t-1}^{\prime}\right)+w\left(\Lambda l_{t}^{\prime}\right)+w\left(l_{t}^{\prime}\right)+w\left(l_{t}^{\prime \prime}\right) \geqslant \\
& \geqslant 1+\rho_{1,2}(\Lambda)+\ldots+\rho_{1,2}(\Lambda)+1=2+(t-1) \rho_{1,2}(\Lambda),
\end{aligned}
$$

поэтому имеет место неравенство

$$
\theta_{1}(C) \geqslant \frac{\rho_{1,2}(\Lambda)(t-1)}{2}+1
$$

Данное неравенство оправдывает важность характеристики $\rho_{1,2}(\Lambda)$ для матрицы $\Lambda$. Подобные неравенства имеют место не только для канонических $X S L$-шифров. Они имеют место для шифров, построенных на основе линейных невырожденных преобразований, в которых отображения (2) являются подстановками на $V_{m}$, например, для схем Фейстеля с $X S L$-схемами в качестве функций усложнения [20].

С точки зрения криптографического синтеза в силу неравенств (23) и (25) предпочтительнее матрицы $\Lambda \in G L(n, 2)$ с высокими коэффициентами рассеивания $\rho_{1,2}(\Lambda)$. Непосредственно из определения (24) следует двойное неравенство

$$
2 \leqslant \rho_{1,2}(\Lambda) \leqslant \varkappa+1
$$


Матрицу $\Lambda \in G L(n, 2)$ называем предельно рассеивающей, если

$$
\rho_{1,2}(\Lambda)=\max _{H \in G L(n, 2)} \rho_{1,2}(H),
$$

и максимально рассеивающей, если $\rho_{1,2}(\Lambda)=\varkappa+1$. При больших $\varkappa$, начиная с определенного зависящего от $m$ значения, максимально рассеивающих матриц не существует.

К максимально рассеивающим матрицам относятся так называемые MDS-матрицы [21], в частности матрицы Коши, используемые в [22]. Нетрудно понять, что свойство максимального рассеивания равносильно невырожденности всех подматриц $\Lambda$ на пересечениях одинакового числа полос и колонок. На это обстоятельство авторам указал А. В. Анашкин. Множество максимально рассеивающих матриц содержит в себе все матрицы с максимальной аддитивной сложностью [23], затрудняющие применение нелинейных обобщений разностного метода [24].

В общем случае вычисление коэффициента рассеивания $\rho_{1,2}(\Lambda)$ по формуле (24) для конкретной матрицы $\Lambda \in G L(n, 2), n=m \varkappa$, является трудоемкой задачей, в связи с чем полезно рассматривать величину $q=\min \min (w(v), w(\Lambda v))$, где внешний минимум берется по тем $v \in V_{n}^{*}$, для которых $w(v)+w(\Lambda v)=\rho_{1,2}(\Lambda), \quad q \leqslant\left\lceil\frac{\varkappa}{2}\right\rceil, 2 q+2 \geqslant \rho_{1,2}(\Lambda) \geqslant 2 q$. Можно также использовать нижние оценки

$$
\rho_{1,2}(\Lambda) \geqslant \min \left\{2(j+1), \min _{i=1,2, \ldots, j}\left\{i+\rho_{1,2}^{(i)}(\Lambda)\right\}\right\}, \quad j=1,2, \ldots,\left[\frac{\varkappa}{2}\right],
$$

где

$$
\rho_{1,2}^{(i)}(\Lambda)=\min _{l \in V_{n}^{*}: w(l)=i} \min \left\{w(\Lambda l), w\left(\Lambda^{-1} l\right)\right\} .
$$

При минимальном $j \in\{1, \ldots,[\varkappa / 2]\}$, для которого

$$
2(j+1) \geqslant \min _{i=1,2, \ldots, j}\left(i+\rho_{1,2}^{(i)}(\Lambda)\right),
$$

имеем

$$
\rho_{1,2}(\Lambda)=\min _{i=1,2, \ldots, j}\left(i+\rho_{1,2}^{(i)}(\Lambda)\right) .
$$

Неравенства (27) позволяют генерировать матрицы $\Lambda \in G L(n, 2)$ с гарантированными нижними оценками показателя $\rho_{1,2}(\Lambda)$ путем их случайного равновероятного выбора из всей совокупности $G L(n, 2)$. Эффективность такого способа подтверждается следующей теоремой. 
Теорема 2. Для $i, h=1,2, \ldots, \varkappa$ при случайном равновероятном выборе матрищы $\Lambda$ из $G L(n, 2)$ выполняется неравенство

$$
\mathbf{P}\left\{\rho_{1,2}^{(i)}(\Lambda) \leqslant h\right\} \leqslant \frac{2\left(\begin{array}{c}
\varkappa \\
\varkappa-h
\end{array}\right)\left(2^{m}-1\right)^{i}\left(\begin{array}{c}
\varkappa \\
i
\end{array}\right)}{2^{m(\varkappa-h)}} .
$$

Доказательство. Пусть $\Lambda$ - произвольная матрица из $G L(n, 2)$, разбитая на $\varkappa$ полос по $m$ строк в полосе и на $\varkappa$ колонок по $m$ столбцов в колонке. Согласно (28) событие $\left\{\rho_{1,2}^{(i)}(\Lambda) \leqslant h\right\}$ выполняется для матриц $\Lambda$ из двух следующих семейств. В первое семейство входят матрицы $\Lambda$, у которых сумма каких-то столбцов, отвечающая умножению $\Lambda$ на вектор-столбец $l \in V_{n}^{*}$ с $w(l)=i$, должна быть нулевой на каких-то $\varkappa-h$ целых группах номеров мощности $m$, по которым производится разбиение матрицы на полосы. (У вектор-столбца $l$ имеется $i$ ненулевых подвекторов из $V_{m}^{*}$.) Второе семейство матриц определяется аналогично, только $\Lambda$ заменяется на $\Lambda^{-1}$. Для доказательства теоремы 2 остается заметить, что фиксированная ненулевая линейная комбинация столбцов случайно равновероятно выбираемой матрицы из $G L(n, 2)$ на фиксированных $H=m(\varkappa-h)$ местах имеет нули с вероятностью $p$, не превосходящей $2^{-H}$. Без ограничения общности в качестве рассматриваемой линейной комбинации можно взять первый столбец. Тогда

$$
p=\frac{\left(2^{n-H}-1\right)\left(2^{n}-2^{1}\right) \cdot \ldots \cdot\left(2^{n}-2^{n-1}\right)}{\left(2^{n}-1\right)\left(2^{n}-2^{1}\right) \cdot \ldots \cdot\left(2^{n}-2^{n-1}\right)}=\frac{2^{n-H}-1}{2^{n}-1} \leqslant \frac{1}{2^{H}} .
$$

Теорема 2 доказана.

В качестве примера использования неравенства (29) при $n=64, m=\varkappa=8$ найдем нижнюю оценку для вероятности

$$
\mathbf{P}\left\{\rho_{1,2}(\Lambda) \geqslant 7\right\}=1-\mathbf{P}\left\{\rho_{1,2}(\Lambda) \leqslant 6\right\} .
$$

Применяя неравенство (27) для $j=3$ к матрицам $\Lambda \in G L(64,2)$ с $\rho_{1,2}^{(1)}(\Lambda) \geqslant 6, \rho_{1,2}^{(2)}(\Lambda) \geqslant 5, \rho_{1,2}^{(3)}(\Lambda) \geqslant 4$, из неравенств $\mathbf{P}\left\{\rho_{1,2}^{(1)}(\Lambda) \leqslant 5\right\}<0.014$, $\mathbf{P}\left\{\rho_{1,2}^{(2)}(\Lambda) \leqslant 4\right\}<0.060, \mathbf{P}\left\{\rho_{1,2}^{(3)}(\Lambda) \leqslant 3\right\}<0.095$, которые следуют из (29), получаем

$$
\begin{aligned}
\mathbf{P}\left\{\rho_{1,2}(\Lambda) \leqslant 6\right\} & \leqslant \mathbf{P}\left\{\rho_{1,2}^{(1)} \leqslant 5\right\}+\mathbf{P}\left\{\rho_{1,2}^{(2)} \leqslant 4\right\}+\mathbf{P}\left\{\rho_{1,2}^{(3)} \leqslant 3\right\} \leqslant \\
& \leqslant 0.014+0.060+0.095<0.17,
\end{aligned}
$$

поэтому $\mathbf{P}\left\{\rho_{1,2}(\Lambda) \geqslant 7\right\}>0.83$. 


\section{3}

Исторически $X S L$-схемам предшествовали $S P$-сети [25-28]. В качестве матриц $\Lambda$ в $S P$-сетях используются подстановочные матрицы, умножением на которые осуществляется перестановка (коммутация) компонент преобразуемых векторов из $V_{n}$ по конкретным подстановкам $P:\{1, \ldots, n\} \rightarrow$ $\{1, \ldots, n\}$. Соответствующее $P$ линейное преобразование $V_{n} \rightarrow V_{n}$ и отвечающую ему матрицу из $G L(n, 2)$ далее обозначаем тоже через $P$.

Для всех подстановочных матриц $P \in G L(n, 2)$ согласно (24) имеем $\rho_{1,2}(P)=2$. Это минимально возможное значение (см. (26)). Таким образом, коэффициент рассеивания $\rho_{1,2}(P)$ не производит ранжирования подстановочных матриц, что следует отнести к недостаткам этой характеристики.

Не в столь «острой» форме этот недостаток имеет место и для разреженных матриц $\Lambda \in G L(n, 2)$, в частности для таких матриц $\Lambda$, что $\Lambda$ и $\Lambda^{-1}$ во всех строках и во всех столбцах имеют одинаковое число единиц, существенно меньшее $n$ [29]. Такие матрицы довольно часто используются при построении алгоритмов блочного шифрования (см. [30-33]).

Семейство показателей $\rho_{1, \tau}(P), \tau \geqslant 2$, определяемое правой частью выражения (22) для канонических $X S L$-шифров с $\tau$ итерациями, также не производит ранжирования подстановочных матриц. Действительно, тогда согласованные системы вектор-столбцов $\mathfrak{L}=\left(\left(l_{i}^{\prime}, l_{i}^{\prime \prime}\right) \in\left(V_{n}^{*}\right)^{2}, i=1,2, \ldots, \tau\right) \in \mathfrak{W}_{1}^{(0)}$ параметризуются (см. п. 1.2) последовательностями

$$
\widehat{\mathfrak{L}}=\left(l_{1}^{\prime}, l_{2}^{\prime}, \ldots, l_{\tau-1}^{\prime}, l_{\tau}^{\prime} ; l_{\tau}^{\prime \prime}\right) \in\left(V_{n}^{*}\right)^{\tau+1},
$$

причем $\mathfrak{L} \in \mathfrak{W}_{1}^{(0)}$ в том и только в том случае, когда

$$
l_{i j}^{\prime}=0 \Leftrightarrow\left(P l_{i+1}^{\prime}\right)_{j}=0, i=1,2, \ldots, \tau-1, j=1,2, \ldots, \varkappa .
$$

Имеет место равенство $\theta_{\mathfrak{L}}=w\left(l_{1}^{\prime}\right)+\ldots+w\left(l_{\tau}^{\prime}\right)$. Обращаясь к выражению (22), получаем

$$
\rho_{1, \tau}(P)=\min _{\mathfrak{L} \in \mathfrak{W}_{1}^{(0)} \backslash\{0\}}\left(w\left(l_{1}^{\prime}\right)+\ldots+w\left(l_{\tau}^{\prime}\right)\right)=\tau \quad \text { для всех } P \in S_{n} .
$$

Тем не менее ранжирование различных коммутаций $P \in S_{n}$ по степени рассеивания, как это формулировалось Шенноном, имело место в практике криптографического синтеза вообще и в упомянутых работах [25-28] в частности. Хорошее рассеивание (по Шеннону) должно исключать применение статистических методов криптографического анализа по коротким фрагментам открытого и соответствующего шифрованного текстов. 
Исходя из этого первоначально степень рассеивания для коммутаций $P$ определялась по лавинному эффекту. Оптимальными в этом отношении, согласно доказываемой ниже теореме 3 , являются подстановки $P \in S_{n}$, для которых переходы $(i, P(i)), i=1,2, \ldots, n$, содержатся среди дуг обобщенных графов де Брейна [34].

Приведем необходимые для формулировки теоремы 3 понятия. Подстановку $\pi: V_{n} \rightarrow V_{n}, x=\left(x_{1}, \ldots, x_{n}\right) \mapsto\left(y_{1}, \ldots, y_{n}\right)=y$ будем называть существенной, если для любых $i, j \in\{1, \ldots, n\}$ существуют такие $x=\left(x_{1}, \ldots, x_{i-1}, x_{i}, x_{i+1}, \ldots, x_{n}\right), x^{\prime}=\left(x_{1}, \ldots, x_{i-1}, x_{i}^{\prime}, x_{i+1}, \ldots, x_{n}\right), x_{i} \neq x_{i}^{\prime}$, что $y_{j} \neq y_{j}^{\prime}$ для $y=\pi(x)$ и $y^{\prime}=\pi\left(x^{\prime}\right)$, причем этим же свойством обладает обратная подстановка $\pi^{-1}$, т. е. для любых $i, j \in\{1, \ldots, n\}$ существуют такие $y=\left(y_{1}, \ldots, y_{j-1}, y_{j}, y_{j+1}, \ldots, y_{n}\right), y^{\prime}=\left(y_{1}, \ldots, y_{j-1}, y_{j}^{\prime}, y_{j+1}, \ldots, y_{n}\right)$, $y_{j} \neq y_{j}^{\prime}$, что $x_{i} \neq x_{i}^{\prime}$. Другими словами, $y_{j}$ существенно зависит от $x_{i}$, a $x_{i}$ существенно зависит от $y_{j}$ для любых $i, j$. Будем называть $S P$-сети с существенными подстановками $\pi$ каноническими $S P$-сетями.

Другое понятие относится к орграфам [34]. Ориентированный граф $Г$ на $n>1$ вершинах называется $\partial$-графом порядка $r \geqslant 1$, если для любых вершин $i, j \in\{1,2, \ldots, n\}$ имеется единственный ориентированный путь из $i$ в $j$, содержащий $r$ дуг. Двойственный к $\partial$-графу $\Gamma$ орграф $\Gamma^{+}$является $\partial$-графом порядка $r+1$. Напомним [35], что вершины $\Gamma^{+}$суть дуги $\Gamma$, и в $\Gamma^{+}$есть дуга из вершины $\alpha$ в вершину $\beta$, если в графе $\Gamma$ конец $\alpha$ совпадает с началом $\beta$. При замене направлений всех дуг в $\partial$-графе $\Gamma$ получаем $\partial$-граф $\bar{\Gamma}$ того же порядка $r$. В работе [34] доказывается, что для $\partial$-графа $\Gamma$ порядка $r \geqslant 1$ на $n>1$ вершинах обязательно $n=m^{r}, m-$ целое, из каждой вершины выходит $m$ дуг, в каждую вершину входит $m$ дуг, имеется ровно $m$ петель. Примерами $\partial$-графов являются графы де Брейна на $n=m^{r}$ вершинах, которые при $r>1$ являются двойственными графам де Брейна на $m^{r-1}$ вершинах.

Формулируемая ниже теорема 3 относится к каноническим $S P$-сетям на $V_{n}, n=m \varkappa$, в которых номера $\{1,2, \ldots, n\}$ для компонент векторов из $V_{n}$ разбиваются на $m$-подмножества

$$
N(j)=\{(j-1) m+1,(j-1) m+2, \ldots, j m\}, \quad j=1,2, \ldots, \varkappa .
$$

Подстановки $\pi$ преобразования $S$ применяются как раз к векторам из $V_{m}$ с номерами компонент из $N(j), j=1,2, \ldots, \varkappa$. Коммутациям $P: V_{n} \rightarrow V_{n}$ далее ставим в соответствие орграф $\Gamma(P)$ на множестве вершин $\{1,2, \ldots, n\}$ с $m$ дугами из каждой вершины $i \in\{1,2, \ldots, n\}$ с концами в множестве $N\left(\left\lceil\frac{P(i)}{m}\right\rceil\right)$, содержащем $P(i)$.

\section{МАТЕМАТИЧЕСКИЕ ВОПРОСЫ КРИПТОГРАФИИ}


Теорема 3. Пусть в канонической $S P$-сети $n=m^{r}, \quad \varkappa=m^{r-1}$, граф $\Gamma(P)$ является д-графом порядка $r$. Тогда подстановка $(S P)^{r}: V_{n} \rightarrow V_{n}-$ существенная.

Прежде чем доказывать эту теорему, заметим, что если граф $\Gamma=\Gamma(P)$ является $\partial$-графом, то он обязательно является двойственным [34], т. е. для некоторого $\partial$-графа $\Gamma_{1}$ порядка $r-1$ на $m^{r-1}$ вершинах имеет место изоморфизм $\Gamma_{1}^{+} \cong \Gamma$. Обратно, если $\Gamma-$ произвольный двойственный $\partial$-граф порядка $r$ на $n=m^{r}$ вершинах, то $\Gamma \cong \Gamma(P)$ для некоторой подстановки $P \in S_{n}$, а вершины можно занумеровать так, что концы дуг с началом в какойлибо вершине образуют одно из $m$-подмножеств $N(j), j \in\{1,2, \ldots, \varkappa\}$. Существование $P \in S_{n}$ здесь следует из того, что каждая вершина находится на ориентированном цикле, длина которого кратна $r$.

Доказательство теоремы 3. Для подстановок $\pi=\pi_{i j}$ используем нумерацию $(i, j) \in\{1,2, \ldots, r\} \times\{1, \ldots, \varkappa\}, \varkappa=m^{r-1}, i$ - номер итерации, $j$ - номер подстановки на $i$-й итерации. Коммутацию $P$ на $i$-й итерации обозначим через $P_{i}, i=1,2, \ldots, r$.

Пусть $\left(y_{1}, \ldots, y_{n}\right)=(S P)^{r}\left(x_{1}, \ldots, x_{n}\right), k, l \in\{1,2, \ldots, n\}$ - произвольные. Покажем, что $y_{l}$ существенно зависит от $x_{k}$, а $x_{k}$ при обратном преобразовании существенно зависит от $y_{l}$.

Преобразование $(S P)^{r}$ представим в виде $r+1$ горизонтальных ярусов из $n$ вершин $\{1,2, \ldots, n\}$. Вершины $i$-го яруса, $i=1,2, \ldots, r$, разбиваем на подмножества $N_{i j}=N(j), j=1,2, \ldots, m^{r-1}$, отвечающие подстановкам $\pi_{i j}$. Из вершины $v$ яруса $i$ проводим дугу в вершину $u$ яруса $i+1$, если $u=P(v), i=1,2, \ldots, r$.

Все вершины множества $N_{1 j_{1}}$, содержащего $k, j_{1}=\left\lceil\frac{k}{m}\right\rceil$, помечаем красным цветом. Далее руководствуемся следующим правилом. Дуги, выходящие из красной вершины, помечаем красным цветом вместе с их концами. Если среди вершин некоторого подмножества $N_{i j}, i=1,2, \ldots, r, j=1,2, \ldots, m^{r-1}$, появилась красная вершина, то все остальные вершины $N_{i j}$ помечаем красным цветом. В результате все $m^{r}$ дуг после $r$-го яруса будут красными, так как граф $\Gamma(P)$ является $\partial$-графом.

Дугу, входящую в вершину $l$ последнего $(r+1)$-го яруса, как и ее начало, пометим фиолетовым цветом. Далее руководствуемся следующим правилом. Если среди вершин некоторого подмножества $N_{i j}, i=1,2, \ldots, r$, $j=1,2, \ldots, m^{r-1}$, оказалась фиолетовая вершина, то все остальные вершины $N_{i j}$ помечаем фиолетовым цветом. Заканчивающуюся фиолетовой вершиной дугу, как и ее начало, помечаем фиолетовым цветом. В результате все $m^{r}$ вершин 1-го яруса будут фиолетовыми, так как граф $\overline{\Gamma(P)}$ является $\partial$-графом. 
Вершины и дуги, помеченные одновременно красным и фиолетовым цветами, объявим зелеными. Так как граф $\Gamma(P)$ является $\partial$-графом порядка $r$, то в каждом ярусе имеется ровно одно подмножество $N_{i j}$ из зеленых вершин, $i=1,2, \ldots, r, j \in\left\{1,2, \ldots, m^{r-1}\right\}$. Аналогично, между ярусами имеется ровно одна зеленая дуга. Иначе в графе $\Gamma(P)$ было бы по меньшей мере два ориентированных пути длины $r$ из вершины $k$ в вершину $l$.

Учитывая последнее замечание, продвигаясь по $S P$-сети снизу вверх, за счет существенности подстановки $\pi$ можно построить векторы

$$
x^{\prime}=\left(x_{1}, \ldots, x_{k-1}, 0, x_{k+1}, \ldots, x_{n}\right) \quad \text { и } \quad x^{\prime \prime}=\left(x_{1}, \ldots, x_{k-1}, 1, x_{k+1}, \ldots, x_{n}\right),
$$

для которых $y_{l}^{\prime} \neq y_{l}^{\prime \prime}$, если $y^{\prime}=(S P)^{r}\left(x^{\prime}\right), y^{\prime \prime}=(S P)^{r}\left(x^{\prime \prime}\right)$. Для построенной пары входов $x^{\prime}, x^{\prime \prime} \in V_{n}$ по фиолетовым дугам будет проходить одно и то же значение бита, а по зеленым дугам - различные значения бит.

Существенная зависимость $x_{k}$ от $y_{l}$ для обратного преобразования доказывается аналогичным образом. Теорема 3 доказана.

\section{4}

Результаты предыдущих пунктов 3.1 - 3.3 показывают, что если и измерять степень рассеивания матриц $\Lambda \in G L(m \varkappa, 2)$, разбитых на $\varkappa$ полос по $m$ строк в полосе и на $\varkappa$ колонок по $m$ столбцов в колонке, с помощью показателей рассеивания зависящей от $\Lambda$ линейной среды $C_{\tau}=C_{\tau}(\Lambda)$ канонических $X S L$-шифров для какого-то числа итераций $\tau$, то необходимо привлекать показатели рассеивания $\theta_{s}\left(C_{\tau}(\Lambda)\right)$, аналогичные (22), но отвечающие многомерному ( $s$-мерному) линейному методу. Используемое при этом определение величины $\theta_{\mathfrak{L}}$ из п. 1.3 должно быть уточнено. В результате возникает 2 -параметрическое семейство коэффициентов рассеивания

$$
\rho_{s, \tau}(\Lambda)=\theta_{s}\left(C_{\tau}(\Lambda)\right), \quad s \geqslant 1, \quad \tau \geqslant 2 .
$$

Коэффициенты $\rho_{1, \tau}, \tau \geqslant 2$, никак не ранжируют подстановочные матрицы. Однако с точки зрения качественного представления о рассеивании наиболее предпочтительными при $n=m^{r}$ являются по теореме 3 коммутации $P \in S_{n}$, для которых $\Gamma(P)$ или $\Gamma\left(P^{-1}\right)$ является $\partial$-графом порядка $r$, в частности графом де Брейна.

Номерами компонент векторов из $V_{n}$ в этом пункте считаем числа $i \in$ $\{0,1, \ldots, n-1\}, n=m^{r}$, представленные в $m$-ичной записи как

$$
\begin{aligned}
& i=\left(i_{0}, i_{1}, \ldots, i_{r-1}\right)=i_{0}+i_{1} m+\ldots+i_{r-1} m^{r-1}, \\
& i_{0}, i_{1}, \ldots, i_{r-1} \in\{0,1, \ldots, m-1\} .
\end{aligned}
$$


Дугами графа де Брейна $\Gamma_{0}$ на множестве $\left\{0,1, \ldots, m^{r}-1\right\}$ являются $\left(i_{0}, i_{1}, \ldots, i_{r-1}\right) \rightarrow\left(i_{1}, \ldots, i_{r-1}, j\right), i_{0}, i_{1}, \ldots, i_{r-1}, j \in\{0,1, \ldots, m-1\}$. При такой нумерации в разбиении

$$
\left\{0,1, \ldots, m^{r}-1\right\}=\bigsqcup_{j=0}^{m^{r-1}-1} N(j)
$$

на $m$-подмножества для $j=\left(j_{0}, j_{1}, \ldots, j_{r-2}\right)$ считаем

$$
\begin{aligned}
N(j) & =\{j m, j m+1, \ldots, j m+m-1\}= \\
& =\left\{\left(i_{0}, j_{0}, j_{1}, \ldots, j_{r-2}\right) \mid i_{0}=0,1, \ldots, m-1\right\} .
\end{aligned}
$$

Тогда $i \in N\left(\left[\frac{i}{m}\right]\right)$.

В рассматриваемой далее канонической $S P$-сети в качестве коммутации $P_{0} \in S_{m^{r}}$, для которой $\Gamma\left(P_{0}^{-1}\right)=\bar{\Gamma}_{0}$, будем брать подстановку $P_{0} \in S_{m^{r}}$, задаваемую равенством

$$
P_{0}(i)=P_{0}\left(i_{0}, i_{1}, \ldots, i_{r-1}\right)=\left(i_{1}, \ldots, i_{r-1}, i_{0}\right) .
$$

Не претендуя на окончательную и единственно возможную характеристику, в качестве показателя рассеивания матрицы $\Lambda \in G L(m \varkappa, 2)$, разбитой на $\varkappa$ полос по $m$ строк и на $\varkappa$ колонок по $m$ столбцов, можно предложить 2-параметрическое семейство коэффициентов рассеивания

$$
\rho_{s, \tau}(\Lambda), \quad s=1,2, \ldots, m, \quad \tau=2, \ldots,\left\lceil\log _{m} n\right\rceil,
$$

с приоритетом для коэффициентов с меньшими параметрами $s, \tau$. Последнее означает, что если при сравнении двух матриц $\Lambda_{1}, \Lambda_{2} \in G L(n, 2)$ с точки зрения их рассеивающих свойств оказалось $\rho_{s, \tau}\left(\Lambda_{1}\right) \neq \rho_{s, \tau}\left(\Lambda_{2}\right)$, то показатели $\rho_{s^{\prime}, \tau^{\prime}}\left(\Lambda_{1}\right)$ и $\rho_{s^{\prime}, \tau^{\prime}}\left(\Lambda_{2}\right), \quad s^{\prime} \geqslant s, \tau^{\prime} \geqslant \tau, \quad\left(s^{\prime}, \tau^{\prime}\right) \neq(s, \tau)$, можно не рассматривать. Для решения вопроса о том, какому из двух коэффициентов $\rho_{s_{1}, \tau_{1}}(\Lambda)$ или $\rho_{s_{2}, \tau_{2}}(\Lambda)$ отдавать предпочтение в случае $s_{1}>s_{2}, \tau_{1}<\tau_{2}$, авторы не располагают какими-либо аргументами.

Верхняя граница $\left\lceil\log _{m} n\right\rceil$ для $\tau$ обосновывается специальными примерами для $n=m^{r}$ и подстановок $P \in S_{m^{r}}$, для которых $(S P)^{r}$ (в отличие от $\left(S P_{0}\right)^{r}$ ) не является существенной подстановкой на $V_{m^{r}}$, но $\rho_{m, \tau}(P)=\rho_{m, \tau}\left(P_{0}\right)$ для $\tau<r$, тогда как $\rho_{m, r}(P)<\rho_{m, r}\left(P_{0}\right)$ (см. теорему 4 ниже). Подобными примерами к обоснованию верхней границы $m$ для $s$ авторы не располагают, не исключено, что и для некоторого $m^{\prime}<m$ справедливо неравенство $\rho_{m^{\prime}, r}(P)<\rho_{m^{\prime}, r}\left(P_{0}\right)$. 
Если бы множество $\left\{(s, \tau) \mid 1 \leqslant s \leqslant m, 2 \leqslant \tau \leqslant\left\lceil\log _{m} n\right\rceil\right\}$ (а может быть, его подмножество) было бы как-то линейно упорядочено (с началом $(1,2)$ и концом $\left.\left(m,\left\lceil\log _{m} n\right\rceil\right)\right)$ по мере уменьшения приоритета $\rho_{s, \tau}(\Lambda)$, то значения

$$
\rho_{s, \tau}(\Lambda), \quad 1 \leqslant s \leqslant m, \quad 2 \leqslant \tau \leqslant\left\lceil\log _{m} n\right\rceil,
$$

можно было бы интерпретировать как цифры после запятой в десятичном представлении вещественного числа. Для неразреженных матриц $\Lambda$ можно обходиться, скорее всего, первой «цифрой» $\rho_{1,2}(\Lambda)$. Необходимость в последующих «цифрах» возникает для разреженных матриц $\Lambda$. Доказываемая ниже теорема 4 утверждает, что в случае подстановочных матриц приходится обращаться даже к самой младшей «цифре» $\rho_{m,\left[\log _{m} n\right\rceil}(\Lambda)$. В ближайшей перспективе желательно выяснить поведение первых из вновь появившихся показателей $-\rho_{2,2}, \rho_{1,3}$ и $\rho_{2,3}$.

При определении показателей рассеивания $\theta_{s}\left(C_{\tau}(\Lambda)\right)$ ограничиваемся тем самым матрицами линейной среды $C_{\tau}(\Lambda)$ для канонических $X S L$-шифров на $\tau \leqslant\left\lceil\log _{m} n\right\rceil$ итераций и значениями $s \leqslant m$. Оставаясь в обозначениях предыдущего пункта, далее предполагаем, что ненулевые матрицы $l_{i j}^{\prime}, l_{i j}^{\prime \prime}$, $i=1,2, \ldots, \tau, j=1,2, \ldots, \varkappa$, размеров $m \times s$ в рассматриваемых согласованных наборах матриц $\mathfrak{L} \in \mathfrak{W}_{s}$ имеют максимально возможный ранг, равный $s$. Без этого предположения в рамках последующих определений имели бы равенство

$$
\theta_{s}\left(C_{\tau}(\Lambda)\right)=\theta_{1}\left(C_{\tau}(\Lambda)\right)
$$

и не получили бы дополнительных возможностей для ранжирования подстановочных и разреженных матриц $\Lambda \in G L(n, 2)$.

Рассматриваемые согласованные наборы матриц

$$
\mathfrak{L}=\left(\left(l_{i}^{\prime}, l_{i}^{\prime \prime}\right), i=1,2, \ldots, \tau\right)=\left(\left(l_{i j}^{\prime}, l_{i j}^{\prime \prime}\right), i=1,2, \ldots, \tau, j=1,2, \ldots, \varkappa\right) \in \mathfrak{W}_{s},
$$

в которых $l_{i}^{\prime \prime}=\Lambda l_{i+1}^{\prime}, i=1,2, \ldots, \tau-1$, параметризуем последовательностями $\widehat{\mathfrak{L}}=\left(l_{1}^{\prime}, l_{2}^{\prime}, \ldots, l_{\tau-1}^{\prime}, l_{\tau}^{\prime} ; l_{\tau}^{\prime \prime}\right)$ из $\tau+1$ матриц размера $n \times s$.

По аналогии с (22) полагаем

$$
\theta_{s}\left(C_{\tau}(\Lambda)\right)=\min _{\mathfrak{L} \in \mathfrak{W}_{s}^{(0)} \backslash\{0\}} \theta_{\mathfrak{L}},
$$

где

$$
\begin{aligned}
\mathfrak{W}_{s}^{(0)} & =\left\{\mathfrak{L} \in \mathfrak{W}_{s} \mid l_{i j}^{\prime}=0 \Leftrightarrow\left(\Lambda l_{i+1}^{\prime}\right)_{j}=0, i=1,2, \ldots, \tau-1, j=1,2, \ldots, \varkappa\right\}, \\
\theta_{\mathfrak{L}} & =\left|\left\{(i, j) \in\{1, \ldots, \tau\} \times\{1, \ldots, \varkappa\} \mid l_{i j}^{\prime} \neq 0\right\}\right| .
\end{aligned}
$$


Теорема 4. Пусть $n=m^{r}, P_{j} \in S_{m^{r}}, j=0,1, \ldots, r-2$,

$$
\begin{aligned}
& P_{j}\left(i_{0}, i_{1}, \ldots, i_{r-j-1}, i_{r-j}, \ldots, i_{r-1}\right)=\left(i_{1}, \ldots, i_{r-j-1}, i_{0}, i_{r-j}, \ldots, i_{r-1}\right), \\
& i_{0}, i_{1}, \ldots, i_{r-1} \in\{0,1, \ldots, m-1\} . \text { Тогда } \\
& \rho_{m, \tau}\left(P_{j}\right)=\tau m^{\tau-1} \text { nри } j+\tau \leq r \quad \text { u } \rho_{m, \tau}\left(P_{j}\right)=\tau m^{r-1-j} n p u j+\tau>r .
\end{aligned}
$$

Согласно теореме 4 имеем $\rho_{m, r-1}\left(P_{1}\right)=\rho_{m, r-1}\left(P_{0}\right)$, но $\rho_{m, r}\left(P_{1}\right)<\rho_{m, r}\left(P_{0}\right)$. Заметим, что подстановка $\left(S P_{0}\right)^{r}$ является существенной, а $\left(S P_{1}\right)^{r}$ таковой не является.

Рассматриваемые в теореме 4 подстановки $P_{0}, P_{1}, \ldots, P_{r-2}$ естественным образом дополняются тождественной подстановкой $P_{r-1}$, для которой утверждение теоремы 4 также справедливо: $\rho_{m, \tau}\left(P_{r-1}\right)=\tau, \tau=2, \ldots, r$.

Подстановки $\left(S P_{j}\right)^{r-j} \in S_{V_{m^{r}}}, j=0,1, \ldots, r-1$, являются существенными на подблоках размера $m^{r-j}$. На каждом из этих $m^{j}$ подблоков подстановки $\left(S P_{j}\right)^{\tau}, \tau \in \mathbb{Z}$, действуют независимо и одинаково. С точки зрения качественного представления о степени рассеивания (по лавинному эффекту) подстановки $P_{0}, P_{1}, \ldots, P_{r-1}$ полностью ранжируются, причем предпочтительнее подстановки $P_{j}$ с меньшими индексами $j$. В то же время

$$
\begin{aligned}
\rho_{m, \tau}\left(P_{r-1}\right) & <\rho_{m, \tau}\left(P_{r-2}\right)<\ldots<\rho_{m, \tau}\left(P_{r-\tau+2}\right)<\rho_{m, \tau}\left(P_{r-\tau+1}\right)< \\
& <\rho_{m, \tau}\left(P_{r-\tau}\right)=\rho_{m, \tau}\left(P_{r-\tau-1}\right)=\ldots=\rho_{m, \tau}\left(P_{1}\right)=\rho_{m, \tau}\left(P_{0}\right) .
\end{aligned}
$$

При $\tau<r$ показатель $\rho_{m, \tau}$ не различает подстановки $P_{0}, P_{1}, \ldots, P_{r-\tau}$, что следует относить к его недостаткам, но при $\tau=r$ все значения

$$
\rho_{m, \tau}\left(P_{j}\right)=\rho_{m, r}\left(P_{j}\right), \quad j=0,1, \ldots, r-2, r-1,
$$

различны, и чем меньше $j$, тем больше этот показатель.

В приводимом далее доказательстве теоремы 4 линейную среду канонической $S P$-сети на $\tau$ итераций с коммутацией $P_{j}, j=0,1, \ldots, r-1$, обозначаем через $C_{j, \tau}$.

Доказательство теоремы 4 достаточно провести для $j=0$. Значение $\tau$ предполагается любым из множества $\{2,3, \ldots, r-1, r\}$.

Как и при доказательстве теоремы 3 , преобразование $\left(S P_{0}\right)^{\tau}$ представляем в виде схемы из $\tau$ горизонтальных ярусов из $m^{r}$ вершин $v \in\left\{0,1, \ldots, m^{r}-1\right\}$ вида $v=\left(v_{0}, v_{1}, \ldots, v_{r-1}\right)=v_{0}+v_{1} m+\ldots+v_{r-1} m^{r-1}$, $v_{0}, v_{1}, \ldots, v_{r-1} \in\{0,1, \ldots, m-1\}$. Вершину $v=\left(v_{0}, v_{1}, \ldots, v_{r-1}\right)$ яруса $j$ (в соответствии с подстановкой $P_{0}$ ) соединяем отрезком с вершиной $\left(v_{1}, v_{2}, \ldots, v_{r-1}, v_{0}\right)$ яруса $j+1, j=1,2, \ldots, \tau-1$. 
На каждом ярусе каждое подмножество $N(j)$ из разбиения (31)

$$
\left\{0,1, \ldots, m^{r}-1\right\}=\coprod_{j \in\left\{0,1, \ldots, m^{r-1}-1\right\}} N(j)
$$

отвечает своей подстановке $\pi \in S_{V_{m}}$ преобразования $S=(\pi, \ldots, \pi), S \in S_{V_{m^{r}}}$. Прибегая к рекомендациям последнего абзаца п. 1.2 , согласованные наборы матриц $\mathfrak{L}=\left(\left(l_{i j}^{\prime}, l_{i j}^{\prime \prime}\right), i=1,2, \ldots, \tau, j=0,1, \ldots, m^{r-1}-1\right), \quad \mathfrak{L} \in \mathfrak{W}_{m}^{(0)} \backslash\{0\}$ будем изображать на описанной схеме из $\tau$ ярусов выделением зеленым цветом всех вершин $N(j)$ из яруса $i$, если $l_{i j}^{\prime} \neq 0, l_{i j}^{\prime \prime} \neq 0$. Ребра с ненулевыми пометками (в виде ненулевых строк из $m$ бит) тоже помечаем зеленым цветом. Условие $\mathfrak{L} \in \mathfrak{W}_{m}^{(0)} \backslash\{0\}$ означает, что:

1) концы зеленых ребер должны быть зелеными,

2) ребро с одним зеленым концом зеленое,

3) если вершина $v \in\left\{0,1, \ldots, m^{r}-1\right\}$ на каком-то ярусе зеленая, то зелеными являются все вершины множества $N\left(\left[\frac{v}{m}\right]\right)$, содержащего $v$.

Если на первом ярусе вершина $\left(v_{0}, v_{1}, \ldots, v_{r-1}\right)$ зеленая, то, проходя по схеме сверху вниз, находим, что согласно условиям 1), 2), 3) зелеными на $j$-м ярусе, $j=1,2, \ldots, \tau$, будут вершины $(*, v_{j}, v_{j+1}, \ldots, v_{r-1}, \underbrace{*, \ldots, *}_{j-1})$.

Здесь предполагается, что на месте $*$ может быть любая «цифра» из множества $\{0,1, \ldots, m-1\}$. В частности, на последнем ярусе будет $m^{\tau}$ зеленых вершин $(*, v_{\tau}, v_{\tau+1}, \ldots, v_{r-1}, \underbrace{*, \ldots, *}_{\tau-1})$.

Проходя теперь по схеме снизу вверх и учитывая условия 1)-3), находим, что на $j$-м ярусе, $j=\tau, \tau-1, \ldots, 2,1$, зелеными вершинами оказываются $(\underbrace{*, \ldots, *}_{\tau-j+1}, v_{\tau}, \ldots, v_{r-1}, \underbrace{*, \ldots, *}_{j-1})$. На каждом ярусе, как видим, имеется ровно

$$
m^{\tau-1}=\frac{m^{\tau-j+1} \cdot m^{j-1}}{m}
$$

подмножеств $N(j)$ из зеленых вершин, поэтому $\rho_{m, \tau}\left(P_{0}\right)=\theta_{m}\left(C_{0, \tau}\right)=\tau m^{\tau-1}$.

При $\tau=r$ все вершины на всех ярусах будут зелеными, поэтому $\rho_{m, r}\left(P_{0}\right)=\theta_{m}\left(C_{0, r}\right)=r m^{r-1}$, а при $\tau>r$ имеем $\theta_{m}\left(C_{0, \tau}\right)=\tau m^{r-1}$. Как раз благодаря последнему обстоятельству для $j>0$ и $\tau>r-j$ имеем $\rho_{m, \tau}\left(P_{j}\right)=\theta_{m}\left(C_{j, \tau}\right)=\tau m^{r-j-1}$.

Теорема 4 доказана.

Авторы благодарны А. М.Зубкову за помощь, оказанную при подготовке работы. 


\section{Список литературы}

[1] Matsui M., "Linear cryptanalysis method for DES cipher”, Lect. Notes Comput. Sci., 765, 1994, 386-397.

[2] Matsui M., "The first experimental cryptanalysis of the Data Encryption Standard", Lect. Notes Comput. Sci., 839, 1994, 1-11.

[3] Kaliski B.S., Robshaw M.J.B., "Linear cryptanalysis using multiple approximations and FEAL”, Lect. Notes Comput. Sci., 1008, 1994, 249-264.

[4] Murphy S., "Overestimates for the gain of multiple linear approximations in symmetric cryptography", IEEE Trans. Inf. Theory, 57 (2011), 4794-4797.

[5] Biryukov A., De Canniere C., Quisquater M., "On multiple linear approximations”, Lect. Notes Comput. Sci., 3152, 2004, 1-22.

[6] Baigneres T., Junod P., Vaudenay S., "How far can we go beyond linear cryptanalysis?", Lect. Notes Comput. Sci., 3329, 2004, 432-450.

[7] Hermelin M., Cho J. Y., Nyberg K., "Multidimentional linear cryptanalysis of reduced round Serpent”, Lect. Notes Comput. Sci., 5107, 2008, 203-215.

[8] Hermelin M., Cho J. Y., Nyberg K., "Multidimentional extension of Matsui's algorithm 2", Lect. Notes Comput. Sci., 5665, 2009, 209-227.

[9] Hermelin M., Multidimentional linear cryptanalysis, PhD Thesis: Aalto Univ. School of Sci. and Technol., 2010, 100 pp.

[10] Hermelin M., Nyberg K., Linear cryptanalysis using multiple linear approximations, Cryptology ePrint Archive, Report 2011/093, 2011.

[11] Малышев Ф.М., “Двойственность разностного и линейного методов в криптографии”, Математические вопросы криптографии, 5:3 (2014), 35-48.

[12] Шеннон К., Работы по теории информации и кибернетике, М.: ИЛ, 1963, 829 с.

[13] Малышев Ф. М., Трифонов Д. И., “Рассеивающие свойства XSLP-шифров”, Математические вопросы криптографии, 7:3 (2016), 47-60.

[14] Большев Л. Н., Смирнов Н. В., Таблицы математической статистики. 3-е изд., М. : Наука, 1983, 416 с.

[15] Broffitt J.D., Randles R.H., "A power approximation for the chi-square goodness-of-fit test: simple hypothesis case", J. Amer. Statist. Assoc., $72: 359$ (1977), 604-607.

[16] Patnaik P. B., "The non-central $\chi^{2}$ and F distributions and their applications", Biometrika, 36 (1949), 202-232.

[17] Pearson E. S., "Note on an approximation to the distribution of non-central $\chi^{2}$ ", Biometrika, 46:3-4 (1959), 364.

[18] Inglot T., "Inequalities for quantiles of the chi-square distribution", Probab. Math. Statist., $\mathbf{3 0 : 2}$ (2010), 339-351.

[19] Двайт Г.Б., Таблицы интегралов и другие математические формулы, М. : Наука, 1978, $223 \mathrm{c}$

[20] Shirai T., Shibutani K., "On Feistel structures using a diffusion switching mechanism", Lect. Notes Comput. Sci., 4047, 2006, 41-56.

[21] Мак-Вильямс Ф. Дж., Слоэн Н. Дж. А., Теория кодов, исправляющих ошибки, М. : Связь, $1979,744 \mathrm{c}$.

2017, T. 8, № 4, С. 29-62 
[22] Youssef A. M., Misters S., Tavares S. E., "The design of linear transformations for substitution permutation encryption networks". In: "SAC'96 Workshop Record", 1997, 40-48.

[23] Малышев Ф. М., “О доле матриц с максимальной аддитивной сложностью”, Дискретная математика, $26: 3$ (2014), 76-78.

[24] Knudsen L.R., "Truncated and higher order differentials", Lect. Notes Comput. Sci., 1008, 1995, 196-211.

[25] Диффи У., Хеллман М., “Защищенность и имитостойкость. Введение в криптографию”, ТИИЭР, $67: 3$ (1979), 71-109.

[26] Kam J. B., Davida G. I., "Structured design of substitution-permutation encryption networks", IEEE Trans. Comput., C-28:10 (1979), 747-753.

[27] Even S., Goldreich O., "On the power of cascade ciphers". In: "Advances in Cryptology: Proceedings of Crypto '83”, N. Y.-London : Plenum Press, 1984, 43-50.

[28] Месси Дж. Л., “Введение в современную криптологию”, ТИИЭР, 76 :5 (1988), 24-42.

[29] Малышев Ф.М., Тараканов В.Е., “О (v, $k)$-конфигурациях”, Матем. сб., 192 :9 (2001), $85-108$.

[30] Kwon D., Kim J., Park S., Sung S. H., Sohn Y., Song J. H., Yeom Y., Yoon E-J., Lee S., Lee J., Chee S., Han D., Hong J., "New block cipher: ARIA", Lect. Notes Comput. Sci., 2971, 2003, 432-445.

[31] Park J., Lee S., Kim J., Lee J., "The SEED encryption algorithm”. In: "Network Working Group. RFC 4009", Reston, Virginia: ISOC, 2005, 17 pp.

[32] Lim C.H., "Specification and analysis of CRYPTON Version 1.0", 1998. http://citeseer.ist.psu.edu.

[33] Панасенко С.П., Алгоритмы шифрования. Специальный справочник, СПб: БХВПетербург, 2009, 578 с.

[34] Малышев Ф. М., Тараканов В. Е., “Обобщенные графы де Брейна”, Матем. заметки, 62 :4 (1997), 540-548.

[35] Холл М., Комбинаторика, М. : Мир, 1970, 421 с. 